ARTICLE

https://doi.org/10.1038/s41467-019-09338-z

\title{
Nucleoside analogue activators of cyclic AMP- independent protein kinase A of Trypanosoma
}

Sabine Bachmaier ${ }^{1}$, Yuri Volpato Santos ${ }^{1}$, Susanne Kramer ${ }^{1,7}$, George Boniface Githure ${ }^{1}$, Thomas Klöckner ${ }^{1}$, Julia Pepperl', Cordula Baums', Robin Schenk1, Frank Schwede [1 2, Hans-Gottfried Genieser², Jean-William Dupuy ${ }^{3}$, Ignasi Forné ${ }^{4}$, Axel Imhof (i) ${ }^{4}$, Jerôme Basquin ${ }^{5}$, Esben Lorentzen ${ }^{6}$ \& Michael Boshart (iD ${ }^{1}$

Protein kinase A (PKA), the main effector of CAMP in eukaryotes, is a paradigm for the mechanisms of ligand-dependent and allosteric regulation in signalling. Here we report the orthologous but CAMP-independent PKA of the protozoan Trypanosoma and identify 7-deazanucleosides as potent activators $\left(E_{50} \geq 6.5 \mathrm{nM}\right)$ and high affinity ligands $\left(K_{\mathrm{D}} \geq 8 \mathrm{nM}\right)$. A cocrystal structure of trypanosome PKA with 7-cyano-7-deazainosine and molecular docking show how substitution of key amino acids in both CNB domains of the regulatory subunit and its unique $\mathrm{C}$-terminal $\alpha \mathrm{D}$ helix account for this ligand swap between trypanosome PKA and canonical cAMP-dependent PKAs. We propose nucleoside-related endogenous activators of Trypanosoma brucei PKA (TbPKA). The existence of eukaryotic CNB domains not associated with binding of cyclic nucleotides suggests that orphan CNB domains in other eukaryotes may bind undiscovered signalling molecules. Phosphoproteome analysis validates 7-cyano-7deazainosine as powerful cell-permeable inducer to explore cAMP-independent PKA signalling in medically important neglected pathogens.

\footnotetext{
${ }^{1}$ Biocenter, Faculty of Biology, Genetics, Ludwig-Maximilians-University Munich (LMU), 82152 Martinsried, Germany. ${ }^{2}$ BIOLOG Life Science Institute, 28199 Bremen, Germany. ${ }^{3}$ Centre de Génomique Fonctionnelle Bordeaux, Université Bordeaux, F-33076 Bordeaux, France. ${ }^{4}$ Biomedical Center, LudwigMaximilians-University Munich (LMU), 82152 Martinsried, Germany. ${ }^{5}$ Max Planck-Institute for Biochemistry, 82152 Martinsried, Germany. ${ }^{6}$ Department of Molecular Biology and Genetics, Aarhus University, Aarhus 8000, Denmark. ${ }^{7}$ Present address: Department of Cell \& Developmental Biology, Biocenter, University of Würzburg, Würzburg 97074, Germany. Correspondence and requests for materials should be addressed to M.B. (email: boshart@lmu.de)
} 
$\mathrm{T}$ he mechanisms of protein kinase regulation, specifically by small second messenger molecules, have been studied in great detail using protein kinase $\mathrm{A}$ (PKA) as a paradigm ${ }^{1}$. PKA, discovered 50 years ago, is present in most eukaryotes except plants, has a highly conserved structure, and is the main effector of the second messenger cAMP. Hence, the synonym cAMP-dependent protein kinase is commonly used. The inactive PKA holoenzyme is a complex of regulatory (PKAR) and catalytic (PKAC) subunits, either as $\mathrm{R}-\mathrm{C}$ heterodimer or $\mathrm{R}_{2}-2 \mathrm{C}$ heterotetramer. The $\mathrm{R}_{2}$ homodimer is formed by an $\mathrm{N}$-terminal dimerization/docking (DD) domain that also mediates subcellular localization via A kinase anchoring proteins (AKAPs). Two C-terminal cyclic nucleotide binding (CNB) domains cooperatively bind two molecules of cAMP, resulting in a conformational change of the $\mathrm{R}$ subunit that releases the active catalytic kinase subunit(s) from the inhibitory pseudo-substrate or substrate site of PKAR. The CNB domain is an ancient evolutionarily conserved domain family with $>7500$ members that confers ligand-dependent allosteric regulation to a diverse range of proteins ${ }^{2}$. In eukaryotes, CNB domains are bound and regulated by cyclic nucleotides. In bacterial transcription factors, some $\mathrm{CNB}$ domains can bind other ligands like heme in the case of the $\mathrm{CO}$ sensing transcription activator $\mathrm{CooA}^{3}$ or chlorinated phenolic compounds in CprK, a member of the ubiquitous CRP-FNR family of transcription activators ${ }^{4}$. In metazoans, PKA has diverse functions ranging from metabolism and gene regulation to development, motility, and memory 5 . Many of these functions are tissue-specific and compartmentalized at the subcellular level ${ }^{6}$. In lower eukaryotes including fungi or apicomplexan protozoa like Plasmodium and Toxoplasma, PKA plays key roles in nutrient sensing, developmental switches, or infectivity processes ${ }^{7-9}$. Most species encode one or two PKAR isoforms and several PKAC isoforms ${ }^{10}$. The resulting holoenzyme isoforms differ in cell typespecificity, developmental expression, sub-cellular localization, and affinity to cAMP, thereby accounting for the pleiotropic functions of cAMP signalling.

Trypanosoma brucei species are kinetoplastid parasites that infect a large variety of mammals, causing severe disease in domestic animals with important economic losses in endemic countries. The parasite is also causative of the deadly human African sleeping sickness, a neglected tropical disease ${ }^{11}$. Transmission is restricted to the habitat of the Tsetse fly in tropical Africa. Development of the parasite in the host and vector is a prerequisite for transmission. This developmental process can be induced by cAMP analogues ${ }^{12-14}$, although this is mediated by intracellular hydrolysis products of these analogues ${ }^{15}$ operating via a complex network of effectors ${ }^{16}$. The parasite has been shown to release cAMP as a mechanism of evading the host's innate immunity ${ }^{17}$. Essential roles of intracellular cAMP signalling have also been documented for cell division ${ }^{12,18-20}$ and social motility ${ }^{21}$. It is therefore surprising that all attempts to detect cAMP-dependent kinase activity in African trypanosomes have failed $22-27$. Genes encoding three PKA catalytic subunit orthologues and one regulatory subunit orthologue have been identified in the T. brucei genome ${ }^{22,26,28}$, whereas alternative cAMP effectors like EPAC orthologues and cNMP-gated ion channels were not detected. By screening a genome-wide RNAi library for cAMP resistance in T. brucei, we identified a novel cAMP binding protein (CARP1) unique to kinetoplastids ${ }^{29}$, yet PKA was not among the hits of the screen. The catalytic subunits of T. brucei PKA are highly conserved with the presence of all 11 canonical kinase subdomains, the essential threonine in the kinase activation loop, and conserved residues implicated in mammalian PKAC's binding to the regulatory PKAR subunits ${ }^{30}$. TbPKAR has a conserved C-terminal part with two CNB domains and the PKA substrate motif (RRTTV) that interacts with and inhibits PKAC.
TbPKAR differs from its metazoan orthologues by an extended $\mathrm{N}$-terminal domain with leucine-rich repeats (LRR) (Fig. 1a). Some amino acid substitutions of consensus residues in the cAMP binding pockets have been noticed in sequence alignments ${ }^{22,31}$. The link between cAMP and PKA remains elusive in Trypanosoma in spite of the excellent overall conservation of the kinase.

There is a surprising deficit of knowledge in signalling mechanisms in these phylogenetically distant organisms and no complete pathway from receptor to effector has been elucidated to date. A possible explanation for this knowledge gap is suggested by the domain architectures found in the kinome of trypanosomes: few known signalling domains or protein-protein interaction domains are linked to the catalytic kinase domains of the 176 identified protein kinases. In addition, unusual domain combinations prevail ${ }^{32}$. Many conserved signalling effectors are likely to be differently connected and wired in various pathway architectures in these phylogenetically distant protozoa. This might also be the case for some second messenger dependencies. Here, we show that Trypanosoma PKA is not a cyclic nucleotidedependent protein kinase. We use a chemical biology approach to identify highly specific activators of Trypanosoma PKA. The first crystal structure of a kinetoplastid PKAR explains the structural requirements for ligand selectivity. We suggest that this PKA has evolved to bind novel ligand(s), possibly taking the role of second messenger(s) in T. brucei. Our new activators are excellent tools to study this cAMP-independent PKA signalling in trypanosomes.

\section{Results}

PKA holoenzymes in Trypanosoma brucei. We first established that the orthologous PKA subunit genes in T. brucei do encode proteins able to form the expected holoenzyme complexes of regulatory $(\mathrm{R})$ and catalytic $(\mathrm{C})$ subunits. One allele of PKAC1 was Tyl-epitope tagged in situ, while the second PKAC1 allele was deleted to generate T. brucei cell line $\Delta c 1 / T y 1-C 1$ (Fig. 1b). The absence of a wild type PKAC1 allele allowed simultaneous detection of the highly similar PKAC2 isoform by a PKAC1/2specific antibody (Fig. 1c and Supplementary Fig. 1a). PKAR was then C-terminally PTP-tagged in situ in cell line $\Delta c 1 / T y 1-C 1$ to generate $\Delta c 1 / T y 1-C 1$ R-PTP (Fig. 1b, c). All three PKA catalytic subunit isoforms were pulled down by PKAR-PTP from lysates of cell line $\Delta c 1 / T y 1-C 1 R$-PTP but not from the control cell line $\Delta c 1 /$ Ty1-C1 (Fig. 1d). Pull down from cell lines expressing Ty1- or HA-tagged PKAC or PKAR subunits independently confirmed the interactions between PKAR and each of PKAC1, 2, 3 in a heterodimeric complex (Supplementary Fig. 1b, c). No coprecipitation of untagged PKAR or other PKAC isoforms was observed with tagged PKAR or PKAC1, 2, or 3 (Fig. 1d and Supplementary Fig. 1c), indicating the absence of a tetrameric $R_{2}$ $2 \mathrm{C}$ complex that is found in mammalian PKA. Heterodimeric PKAR-C complexes are not unusual in lower eukaryotes ${ }^{27,33,34}$.

The catalytic function of the kinase is essential for growth and viability, as RNAi-mediated repression of the catalytic subunits PKAC1/2 or PKAC3 is lethal or growth inhibitory, respectively ${ }^{35}$ (Supplementary Fig. 2). Severe cell division defects are detected, as cytokinesis stages and multinucleated cells accumulate. This phenotype is frequently found when targeting essential trypanosome genes ${ }^{36}$. It does not necessarily indicate a specific role in cytokinesis. RNAi-mediated repression of PKAR has a very similar phenotype due to the rapid decrease of free PKAC1 (Supplementary Fig. 2). This indicates degradation of free PKAC released upon holoenzyme dissociation, as was observed for PKAC in mammalian cells ${ }^{37}$. Nevertheless, cell clones can be selected that maintain a basal PKAC1 level sufficient for survival 
a

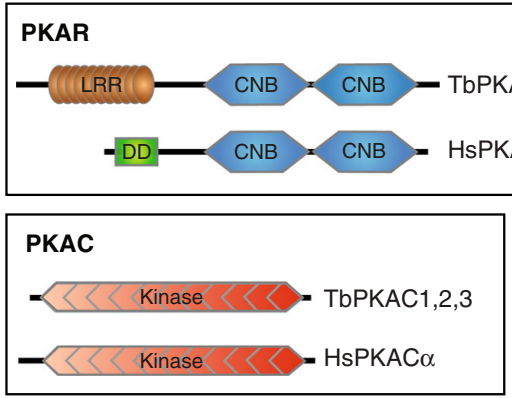

b

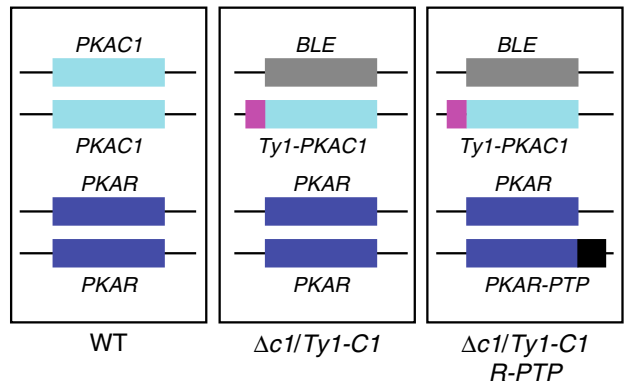

C

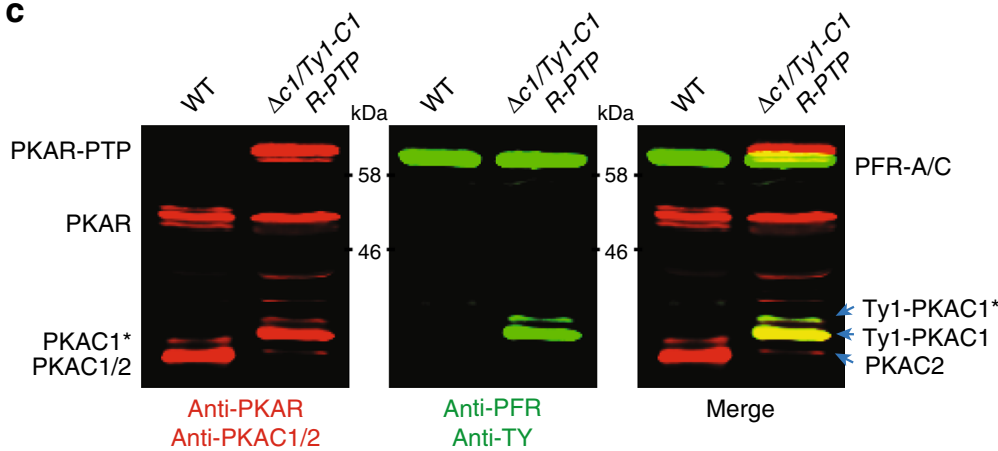

d
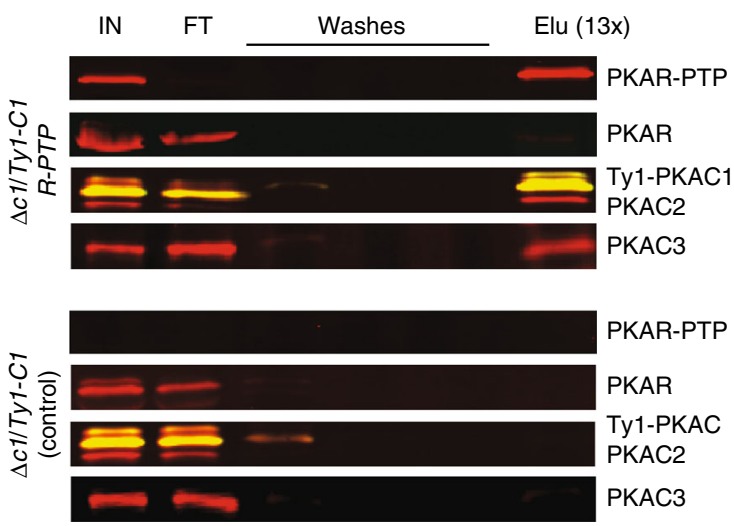

Fig. 1 PKA holoenzyme complexes in T. brucei. a Domain architecture of PKAR (top) and PKAC (bottom) orthologues from T. brucei (Tb) (TriTrypDB accessions: PKAR, Tb927.11.4610; PKAC1, Tb927.9.11100; PKAC2, Tb927.9.11030; PKAC3, Tb927.10.13010) compared to human (Hs) PKA (Uniprot accessions: PKARI $\alpha$, P10644; PKAC $\alpha$, P17612). LRR leucine-rich repeat region, DD dimerization/docking domain, CNB cyclic nucleotide binding domain, kinase kinase domain. b Genotypes of cell lines with in situ tagged PKAC1 (Ty1-C1, cyan; Ty1 epitope tag in magenta) and PKAR (R-PTP, blue; PTP-tag in black) compared to wild type (WT). The phleomycin resistance cassette (BLE, grey) is indicated. c Two-colour fluorescent western blot analysis of the double tagged cell line ( $\triangle C 1 /$ Ty1-C1 R-PTP) using anti-PKAR and anti-PKAC1/2 (left panel, red signals), and anti-Ty1 and anti-PFR-A/C (loading control) (middle panel, green signals). The merge of both channels is shown on the right panel. Ty1 causes a mobility shift of PKAC1 and enables the detection of PKAC2 in cell lines devoid of wild type PKAC1. Note that PKAC1 and PKAR appear as doublet bands that we interpret as modification (PKAC1*) and allelic polymorphism, respectively, in the MiTat 1.2 cell line. d PTP affinity purification followed by western blot analysis of double tagged and control cell lines using antibodies as in c and anti-PKAC3. Equivalent amounts of soluble input material (IN), flow-through (FT), washes, and 13 equivalents of the eluate (Elu) were loaded. Source data to $\mathbf{c}$ and $\mathbf{d}$ are provided as a Source Data file

after homozygous deletion of PKAR. The resulting $\triangle p k a r / \triangle p k a r$ cell lines ${ }^{38}$ used later in this work show a mild growth phenotype with population doubling time (PDT) of $7.5 \mathrm{~h}$ versus $5.4 \mathrm{~h}$ for wild type cells.

Trypanosoma PKA is not activated by cAMP. The PKA holoenzymes were immunoprecipitated from trypanosomes expressing epitope-tagged PKA subunits to assay PKA activity. Contaminating kinase activities in the precipitate were excluded as (1) Ty1-affinity purification from wild type cells or cells expressing a catalytically inactive Ty1-PKAC1 N153A mutant ${ }^{39}$ did not pull down PKA-specific activity and (2) phosphorylation of the PKA-specific substrate kemptide was inhibited by the PKAspecific pseudo-substrate peptide PKI(5-24) ${ }^{40}$ (Supplementary Fig. 3a). The basal activity of holoenzymes immunoprecipitated from cells expressing PKAR-Ty1 or Ty1-PKAC1 or HA-PKAC2 was not increased by cAMP (Supplementary Fig. 3b), even when the cyclic nucleotide was added at unphysiologically high concentrations $(1 \mathrm{mM})$. In the same experiments, cGMP activated at $\geq 100 \mu \mathrm{M}$ (Supplementary Fig. 3b). Some activation by cGMP had been noted before ${ }^{22}$, but several lines of evidence argue against 
the presence of cGMP or cGMP signalling in trypanosomatids ${ }^{41}$, minimizing the likelihood that cGMP is a physiological activator of the kinase. The unconventional cyclic nucleotides cXMP, cIMP, cCMP, and cUMP also did not show any significant effects (Supplementary Fig. 3c). In order to exclude unsuitable assay conditions, an in vivo assay for PKA activity was established that is based on transgenic expression of the heterologous PKA substrate VASP (human platelet vasodilator stimulated phosphoprotein) in T. brucei. Phosphorylation of VASP at the PKA site Ser-157 causes an electrophoretic mobility shift from 46 to 50 $\mathrm{kDa}^{42}$ (Supplementary Fig. 3e). Non-phosphorylated $(46 \mathrm{kDa})$ and phosphorylated VASP $(50 \mathrm{kDa})$ were quantified by western blot analysis. The ratiometric determination of VASP phosphorylation (non-phosphorylated/phosphorylated) is a reliable proxy for PKA activity. The myristoylated membrane-permeable peptide inhibitor myr-PKI(14-22) ${ }^{40}$ reduced the measured activity to background (Supplementary Fig. 3f). We then examined the effects of cAMP on PKA activity in live cells by three independent approaches. First, pharmacological elevation of intracellular cAMP was accomplished by inhibition of phosphodiesterases (PDEs) with a highly potent inhibitor of trypanosomal PDEs (CpdA, now renamed as NPD-001). The intracellular cAMP content increased up to 600 -fold upon treatment with CpdA (Fig. 2a) but did not elicit any change in in vivo PKA activity (Fig. 2b). Second, reverse genetic elevation of intracellular cAMP was achieved by inducible RNAi-mediated depletion of the major cAMP-specific PDEs, PDEB1 and PDEB2 ${ }^{20}$. The resulting 45-fold increase of cAMP content (Fig. 2c) also did not stimulate PKA activity (Fig. 2d). Third, VASP expressing cells were treated with membrane-permeable cAMP analogues 8-pCPT-cAMP, 8pCPT-2'-O-Me-cAMP, or cAMP-AM (see Supplementary Table 1). The latter is a prodrug cleaved by esterases to deliver cAMP intracellularly ${ }^{43}$. No PKA activation was detected for any of the analogues up to $1 \mathrm{mM}$ (Supplementary Fig. 3h). Membrane-permeable cGMP derivatives also had no effect on PKA activity in vivo (Supplementary Fig. 3i).

The drug dipyridamole induces PKA activity in trypanosomes. The most compelling evidence against cAMP-dependent PKA activity in trypanosomes is the lack of any change in kinase activity upon an up to 600-fold increase in intracellular cAMP content, caused by the PDE inhibitor CpdA. Prior to the availability of CpdA, the same experiment was done with dipyridamole, a potent inhibitor of mammalian $\mathrm{PDEs}^{44}$ with modest activity against trypanosomal PDEs ${ }^{15,45}$. Initially, we were misled by a dose- and time-dependent induction of VASP phosphorylation by dipyridamole (Fig. 2e, f, Table 1) that correlated with a moderate increase in intracellular cAMP content (Fig. 2g). As the data shown in Fig. 2a-d provide strong evidence that this effect by dipyridamole cannot be caused by the increase in cAMP, dipyridamole must induce PKA activity either directly or indirectly by a cAMP-independent mechanism. Yet, the effect is clearly mediated by PKAR, as no induction was detected in a VASP reporter cell line with homozygous deletion of PKAR (Fig. 2h).

Compound screening for activators of TbPKA. Dipyridamole, a licensed drug inhibiting thrombocyte aggregation, is a PDE inhibitor but also interferes with adenosine transport and metabolism ${ }^{46}$. The possible link between PKA activation and purine metabolism motivated a targeted compound screen. We tested 13 different purine nucleoside or nucleotide analogues (Supplementary Table 1) for activity in the in vivo PKA reporter assay. Most of them are predicted to be membrane permeable due to lipophilic groups. Out of these, the four 7-deazaadenosine (tubercidin, Tu) analogues toyocamycin (Toyo), 5-iodo- tubercidin (5-I-Tu), 5-bromo-tubercidin (5-Br-Tu), and sangivamycin induced PKA activity with $\mathrm{EC}_{50}$ values of $88 \mathrm{nM}, 390$ $\mathrm{nM}, 625 \mathrm{nM}$, and $39 \mu \mathrm{M}$, respectively (Fig. 3a, Table 1). The remaining compounds had either a slight inhibitory (8-pCPTAdo, 8-pCPT-2-'O-Me-Ado) or no effect (Tu, 8-pCPT-Guo, 2Cl-Ado, 8-pCPT-Ade, 6-Br-Tu, 8-Br-Ado, 6-Cl-PuR) in the PKA reporter assay (Fig. 3, Supplementary Fig. 4a). The most potent activator, toyocamycin, did not induce phosphorylation of VASP in the pkar knock out genetic background (Supplementary Fig. 4b), demonstrating that PKA is the target kinase. Growth or viability of the parasites was affected by continuous treatment with all 7-deazaadenosine analogues (Table 1). However, this was apparently due to off-target effects, as the drugs had very similar effects on pkar knock out cells (Table 1). Since these analogues are known to target multiple proteins and processes in mammalian cells, including kinases and synthesis of DNA, RNA, and proteins (reviewed in ref. ${ }^{47}$ ), growth or viability phenotypes were expected.

7-Deazaadenosine analogues bind and activate TbPKAR. The identified compounds may activate trypanosome PKA either directly or indirectly in the cell-based reporter assay. To address this, tagged PKAR and PKAC1 subunits were co-expressed in the heterologous Leishmania tarentolae expression system and the holoenzyme complex was isolated by tandem affinity purification (Supplementary Fig. 4e, inset). In vitro kinase assays with kemptide and $\left[{ }^{32} \mathrm{P}\right]-\mathrm{ATP}$ as substrates showed kinase activation upon addition of tubercidin and 5-substituted tubercidin analogues with the following order of potency: 5-I$\mathrm{Tu}>$ Toyo $>5-\mathrm{Br}-\mathrm{Tu}>$ tubercidin $>$ sangivamycin (Fig. 3a, Table 1). The $\mathrm{EC}_{50}$ values measured with recombinant $T$. brucei holoenzyme purified from L. tarentolae were in the same order of magnitude as the $\mathrm{EC}_{50}$ values of the in vivo $\mathrm{PKA}$ reporter assay. Up to 3.4-fold differences between in vitro kinase assay and cell-based assay are likely due to uptake, accumulation, or metabolism of the individual compounds in trypanosome cells. Tubercidin enters the trypanosome cell via nucleoside transporters $^{48}$, while the 5 -substituted analogues are more lipophilic and predicted to cross the cell membrane by passive diffusion. Tubercidin uptake might be too slow to cause PKA activation within the time frame of the in vivo reporter assay. The evidence for a direct mode of action provided by in vitro kinase assays was further corroborated by measuring binding parameters between the activating compounds and purified $\mathrm{N}$ terminally truncated T. brucei PKAR(199-499) expressed in Escherichia coli (Supplementary Fig. 4h). Isothermal titration calorimetry (ITC) determined a $K_{\mathrm{D}}$ of $57 \mathrm{nM}$ for toyocamycin with a ligand to protein molar ratio of $2.1( \pm 0.2)$, indicating availability of both CNB sites for binding (Fig. 3a, Table 1). A slightly lower $K_{\mathrm{D}}$ of $32 \mathrm{nM}$ was obtained for $5-\mathrm{I}-\mathrm{Tu}$ with a ligand to protein molar ratio of only $1.2( \pm 0.2)$. We suggest that $5-\mathrm{I}-\mathrm{Tu}$ has a preference for one binding site, whereas the $K_{\mathrm{D}}$ value for Toyo averages over both binding sites. This is supported by the different thermodynamic signatures that indicate enthalpically-driven binding, which for 5-I-Tu is counteracted by a negative entropic effect (Fig. 3c). It should be noted that all binding assays are performed with the ligand-free (apo) form of the PKAR subunit, whereas kinase assays probe the R-C holoenzyme. Nevertheless, the binding $K_{\mathrm{D}}$ values for the analogues are close to the respective $\mathrm{EC}_{50}$ for kinase activation in vitro. Most importantly, the values for Toyo and 5-I-Tu are similar to the $K_{\mathrm{D}}$ for cAMP binding to mammalian PKARIa measured under identical conditions $(28 \pm 5 \mathrm{nM}$, Supplementary Fig. $4 \mathrm{f}$ and ref. ${ }^{49}$ ). Dipyridamole, whose activation of $T$. brucei PKA had initially guided our compound screen, did not 

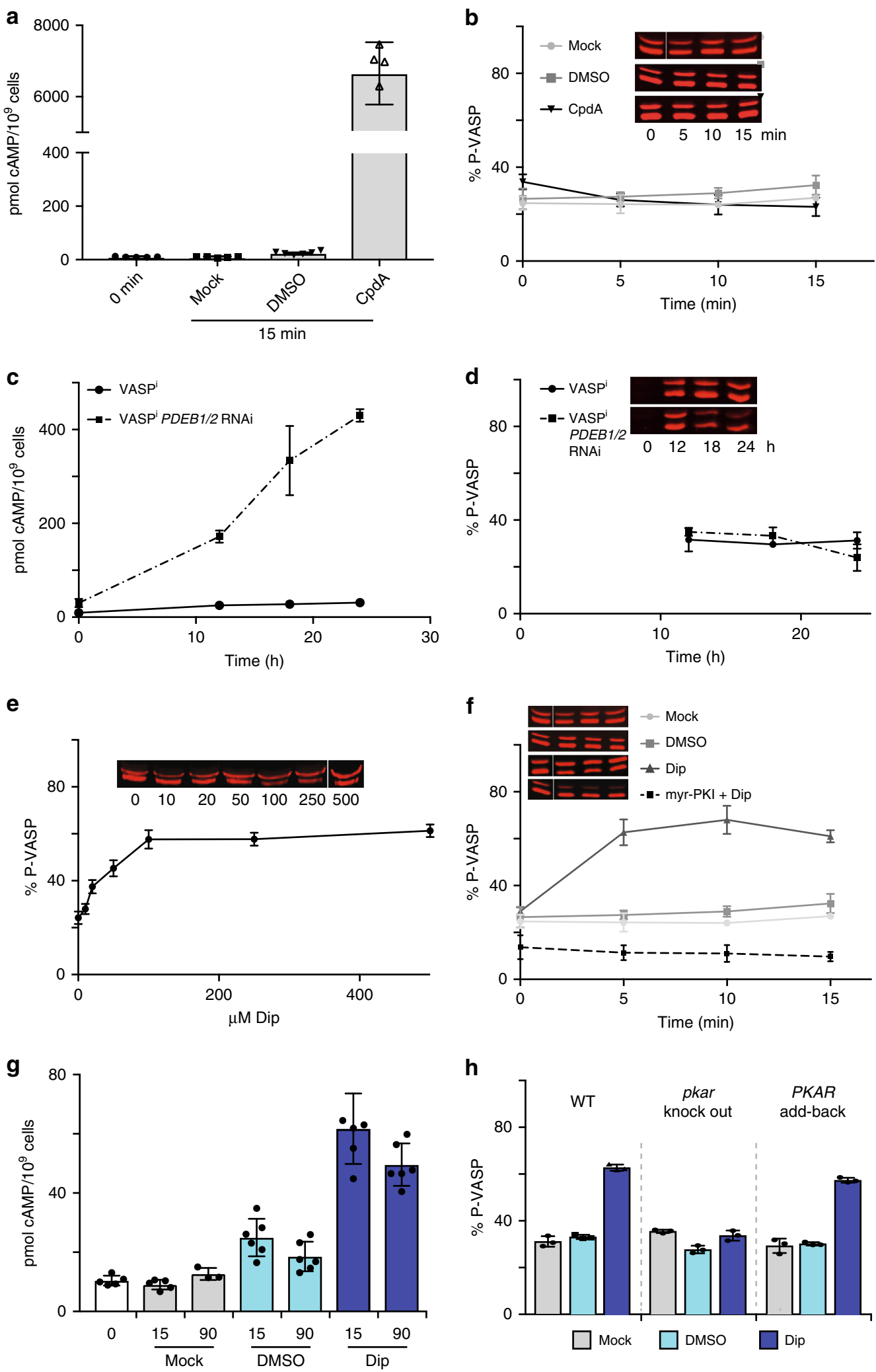

h

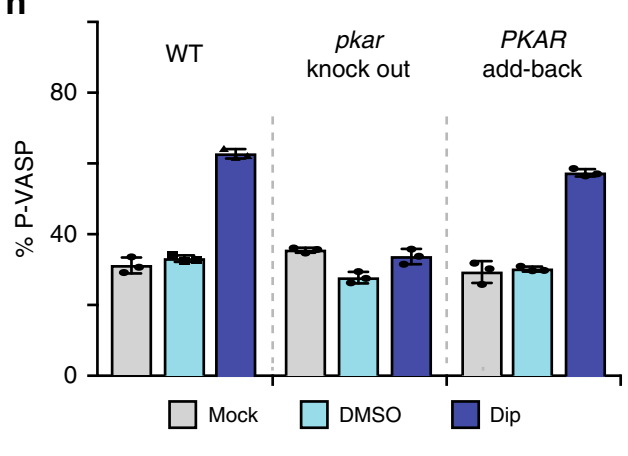

have any effect in the in vitro kinase assay (Supplementary Fig. 4e), supporting an indirect mode of action in vivo.

Development of a trypanosome-specific PKA activator. In mammalian cells, some nucleoside analogues have been reported to be significantly less toxic when the adenosine moiety is replaced by inosine $e^{50,51}$. Therefore, as the first step in optimization, we changed the purine ring side groups of toyocamycin (7cyano-7-deazaadenosine) to match inosine, resulting in the compound 7-cyano-7-deazainosine (7-CN-7-C-Ino). Compared to Toyo, 7-CN-7-C-Ino had 36-fold higher activation potency on the purified holoenzyme $\left(\mathrm{EC}_{50} 6.5 \pm 4.6 \mathrm{nM}\right)$ and 7 -fold higher binding affinity to PKAR $\left(K_{\mathrm{D}} 8 \pm 2 \mathrm{nM}\right)$ in ITC measurements 
Fig. 2 T. brucei PKA is not activated by cAMP. a Intracellular [CAMP] (mean \pm SD of independent replicates; $n=5$ ( 0 min, Mock); $n=6$ (DMSO, CpdA)) and $\mathbf{b}$ in vivo PKA activity (with western blot inset) in cells treated or not (Mock) for 15 min with the PDE inhibitor CpdA ${ }^{19}$ (10 $\mu$ M; now renamed as NPD$001^{74}$ ) or solvent (1\% DMSO). Release of cAMP into the medium upon treatment was neglectable (Supplementary Fig. 3d). c Intracellular [cAMP] (mean \pm SD of $n=3$ independent replicates) and $\mathbf{d}$ in vivo PKA activity (with western inset) upon inducible $\left(1 \mu \mathrm{g} \mathrm{ml} \mathrm{l}^{-1}\right.$ tetracycline) RNAi repression of PDEB1 and PDEB2. Kinase activity cannot be determined at time 0 , since the RNAi cell line (VASPi PDEB1/2 RNAi) and the control line (VASPi) both harbour a tetracycline inducible VASP transgene. e Dose response of in vivo PKA activity upon 15 min treatment with dipyridamole (Dip). $\mathbf{f}$ Time course of in vivo PKA activity upon treatment with $100 \mu \mathrm{M}$ dipyridamole (Dip) or dipyridamole + myr-PKI(14-22) or solvent (1\% DMSO) or Mock. $\mathbf{g}$ Intracellular [cAMP] (mean \pm SD of independent replicates; $n=5$ ( 0 min, Mock 15 min); $n=3$ (Mock 90 min); $n=6$ (DMSO, Dip)) upon treatment with $100 \mu M$ dipyridamole (Dip) or $1 \%$ solvent (DMSO) or Mock for 15 or 90 min. $\mathbf{h}$ In vivo PKA activity upon treatment as in $\mathbf{g}$ for 15 min in wild type (WT), homozygous pkar knock out and PKAR add-back (in situ rescue) cells. For all in vivo kinase reporter assays, one representative western blot is shown as inset and data points are mean \pm SD of $n=3$ independent replicates. Source data are provided as a Source Data file

Table 1 Summary of TbPKA activators

\begin{tabular}{|c|c|c|c|c|c|c|}
\hline Compound & $\begin{array}{l}\mathrm{EC}_{50} \text { in vivo PKA } \\
\text { assay }^{\mathrm{a}}\end{array}$ & $\begin{array}{l}\mathrm{EC}_{50} \text { in vitro PKA } \\
\text { assayb }\end{array}$ & $\begin{array}{l}K_{D} \text { in vitro binding to } \\
\text { PKAR (ITC) }\end{array}$ & $\begin{array}{l}\text { Molar ratio of } \\
\text { binding (ITC) }\end{array}$ & $\begin{array}{l}\text { IC }_{50} \text { growth } \\
\text { WT }^{\text {d }}\end{array}$ & $\begin{array}{l}I_{50} \text { growth pkar } \\
\text { KO }^{\text {d }}\end{array}$ \\
\hline 7-CN-7-C-Ino & $838 \pm 139$ nM & $6.5 \pm 4.6 \mathrm{nM}$ & $8 \pm 2 n M$ & $2 \pm 0.2$ & $\geq 125 \mu \mathrm{M}$ & $\geq 125 \mu \mathrm{M}$ \\
\hline Toyo & $87.6 \pm 9.6 \mathrm{nM}$ & $234 \pm 92 \mathrm{nM}$ & $57 \pm 25 \mathrm{nM}$ & $2.1 \pm 0.1$ & $739 \pm 59 n M$ & $690 \pm 58 \mathrm{nM}$ \\
\hline 5-I-Tu & $390 \pm 20$ nM & $203 \pm 79$ nM & $32 \pm 9 \mathrm{nM}$ & $1.2 \pm 0.2$ & $330 \pm 38 \mathrm{nM}$ & $346 \pm 41 \mathrm{nM}$ \\
\hline $5-\mathrm{Br}-\mathrm{Tu}$ & $625 \pm 66 \mathrm{nM}$ & $1.7 \pm 1.4 \mu \mathrm{M}$ & n.d. & n.d. & $874 \pm 122 \mathrm{nM}$ & $1009 \pm 95 \mathrm{nM}$ \\
\hline Tubercidin & No effect & $5.0 \pm 0.5 \mu \mathrm{M}$ & n.d. & n.d. & n.d. & n.d. \\
\hline Sangivamycin & $39.3 \pm 5.6 \mu \mathrm{M}$ & $10.8 \mu \mathrm{M}$ & n.d. & n.d. & $713 \pm 40 \mathrm{nM}$ & $1548 \pm 171 \mathrm{nM}$ \\
\hline Dipyridamole & $22.4 \pm 4.2 \mu \mathrm{M}$ & No effect & n.d. & n.d. & $9.9 \pm 1 \mu \mathrm{M}$ & $16.3 \pm 1.8 \mu \mathrm{M}$ \\
\hline \multicolumn{7}{|c|}{ 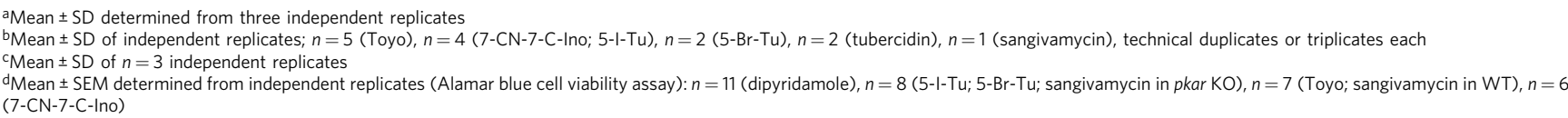 } \\
\hline
\end{tabular}

(Fig. 3b, Table 1). The increased affinity was mainly due to gain in the entropic component of binding $\left(-T \Delta S=-2.3 \mathrm{kcal} \mathrm{mol}^{-1}\right.$ versus $-0.9 \mathrm{kcal} \mathrm{mol}^{-1}$ for Toyo) (Fig. 3c). This surprising increase in activation potency upon introduction of a single structural modification did not, however, translate into increased potency in the in vivo kinase reporter assay $\left(\mathrm{EC}_{50} 838 \pm 139 \mathrm{nM}\right)$, probably due to slower uptake or faster intracellular metabolism of the 7-deazainosine analogue. In a cell line with homozygous deletion of PKAC3 and simultaneous RNAi depletion of PKAC1/2 (Supplementary Fig. 4d), phosphorylation of the PKA reporter substrate VASP was undetectable with or without treatment by 7 CN-7-C-Ino (Supplementary Fig. 4c). This control fully corroborated the PKA specificity of the in vivo reporter assay based on VASP Ser-157 phosphorylation in trypanosomes. The cytotoxicity of 7-CN-7-C-Ino was drastically reduced compared to Toyo (>170-fold increase of $\mathrm{EC}_{50}$ for cell viability, Table 1).

7-CN-7-C-Ino, Toyo, or 5-I-Tu did not bind to purified human PKARIa, whereas CAMP binding to the same PKARIa preparations $\left(K_{\mathrm{D}} 28 \mathrm{nM}\right.$, Supplementary Fig. $\left.4 \mathrm{f}\right)$ was intermediate between the affinities of CNB-A and CNB-B that have been measured before separately ${ }^{52}$. Consistently, Toyo did not activate the mammalian PKARIa $\mathrm{P}_{2}-2 \mathrm{PKACa}$ holoenzyme, even at concentrations 270 -fold above the $\mathrm{EC}_{50}$ for the T. brucei PKA holoenzyme (Supplementary Fig. 4g). As expected from the kinase assays (see Fig. 2b, d and Supplementary Fig. 3b), recombinant $T$. brucei PKAR did not bind cAMP (Supplementary Fig. 4h). In summary, a potent, highly specific, and nontoxic activator of the cAMP-independent trypanosomal PKA has been developed.

Crystal structure of parasite PKAR with 7-CN-7-C-Ino. To explore the molecular basis of binding and activation selectivity of Trypanosoma PKA for 7-deaza nucleoside analogues, we cocrystallized PKAR with 7-CN-7-C-Ino. The highest resolution X- ray diffraction data were obtained using truncated PKAR (200-503) of the related parasite Trypanosoma cruzi (Supplementary Fig. 5, see Supplementary Table 2 for data collection and refinement statistics) that is highly homologous in primary sequence (79\% identity) and structural alignment to the T. brucei PKAR fragment. The protein adopts a dumbbell-like structure (Fig. 4a) consisting of two 8-stranded beta barrels (CNB-A and CNB-B) linked by an alpha helical element (R337 to N361, three helices). A 7-CN-7-C-Ino molecule can be clearly recognized on top of each beta barrel by the electron density in the Fo-Fc omit map (Fig. 4b, c). In bovine PKAR (PDB 1RGS), the CNB-A and $\mathrm{CNB}-\mathrm{B}$ domains are linked via allosteric communication ${ }^{30}$. When a cAMP molecule enters the CNB-B of PKAR, it is capped by a hydrophobic residue (Y371) via $\pi$-stacking interaction. This event triggers the rupture of a salt bridge (E261-R366) and consequently destabilizes the holoenzyme conformation. Tryptophan W260 thereby moves $30 \AA$ closer to CNB-A where it caps the second cAMP molecule in that binding site by an analogous $\pi$ stacking interaction. The conformational change releases an active C-subunit. The amino acids taking part in these sequential ligand binding events linked to holoenzyme activation are readily identified in 7-CN-7-C-Ino-bound T. cruzi PKAR by alignment with cAMP-bound bovine PKARIa (Fig. 4a, Supplementary Movie 1). In TcPKAR(200-503) the capping residues in CNB-A and $-\mathrm{B}$ are $\mathrm{Y} 371$ and $\mathrm{Y} 483$, respectively, while a salt bridge might be formed by the pair R476-E372. Despite only 35\% sequence identity, the $\mathrm{C}_{\alpha}$ alignment of both structures shows very high conservation (RMSD, root mean square deviation $=3.25 \AA$ ) including the fragmented $\alpha \mathrm{B} / \mathrm{C}$ helix (Supplementary Fig. 6, Supplementary Movie 2) that is key of the conformation change mediating allosteric activation of bovine PKARIa ${ }^{30}$.

Both CNB-A and -B pockets are occupied by 7-CN-7-C-Ino with the ribose moiety in a position very similar to the position of the ribose moiety of cAMP bound to bovine PKARIa (Fig. 4a, 
a

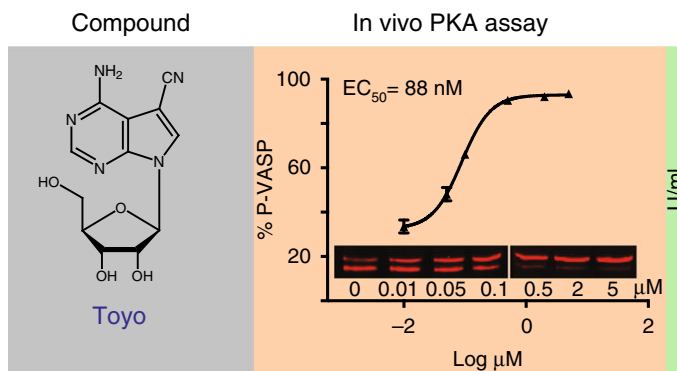

(1)

5-I-Tu

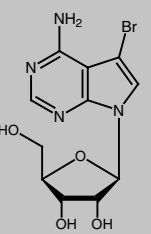

5-Br-Tu

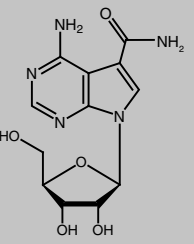

Sangivamycin

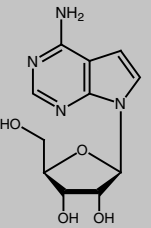

Tubercidin
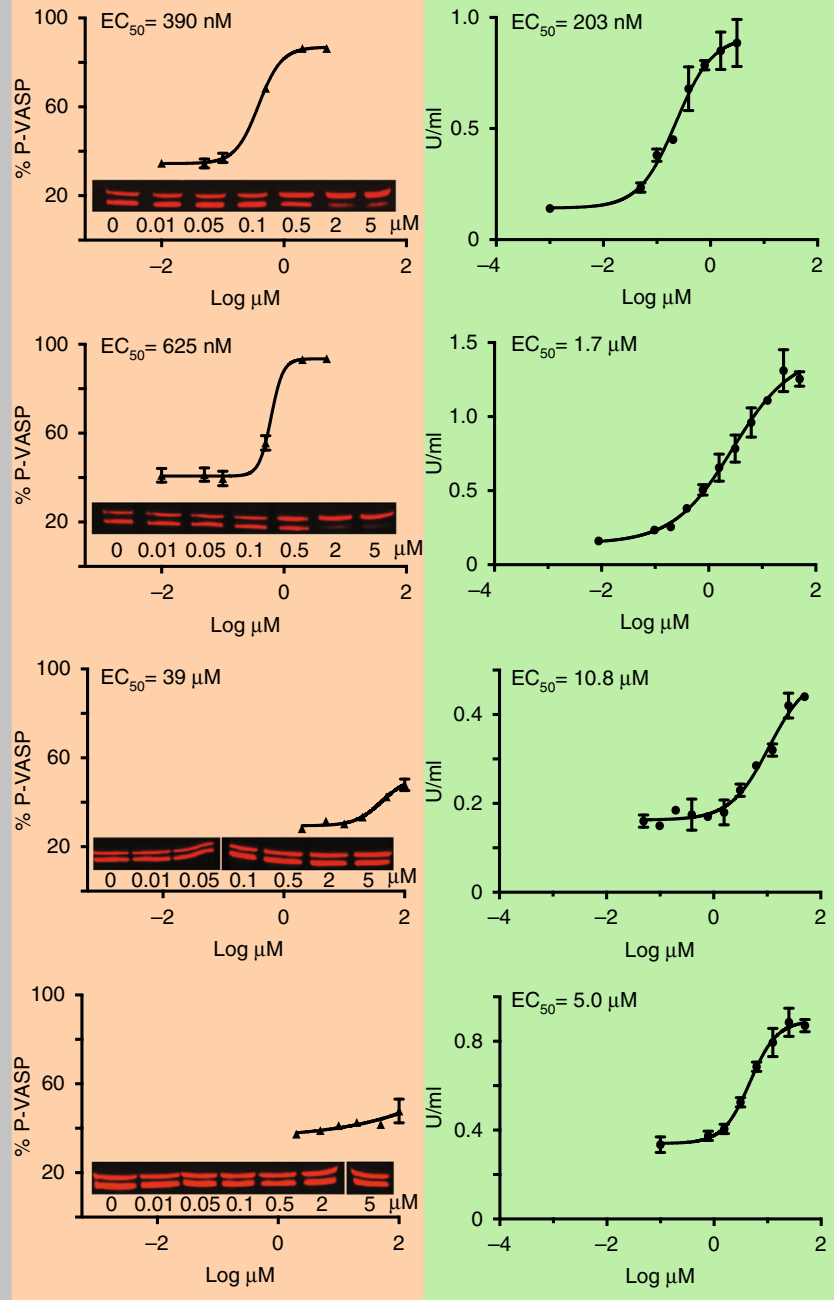

Binding to R subunit (ITC)
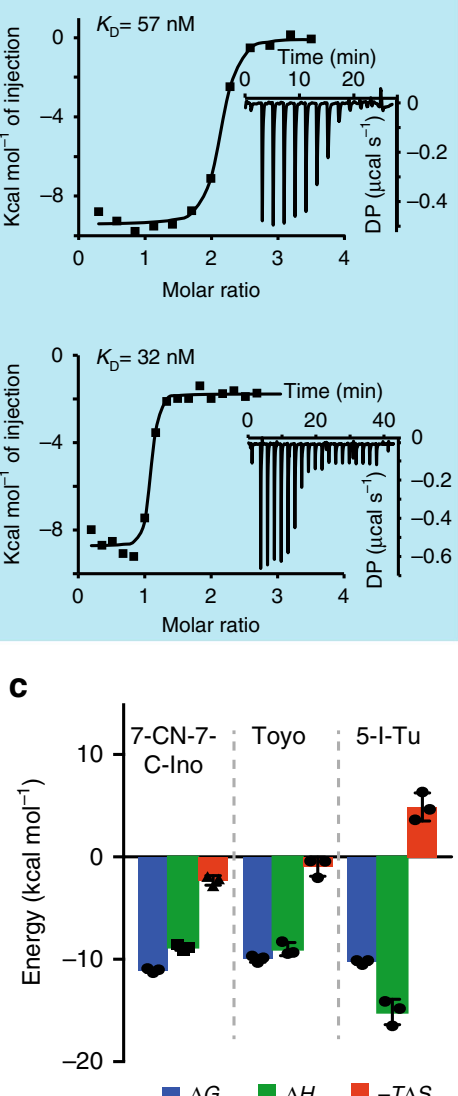

b

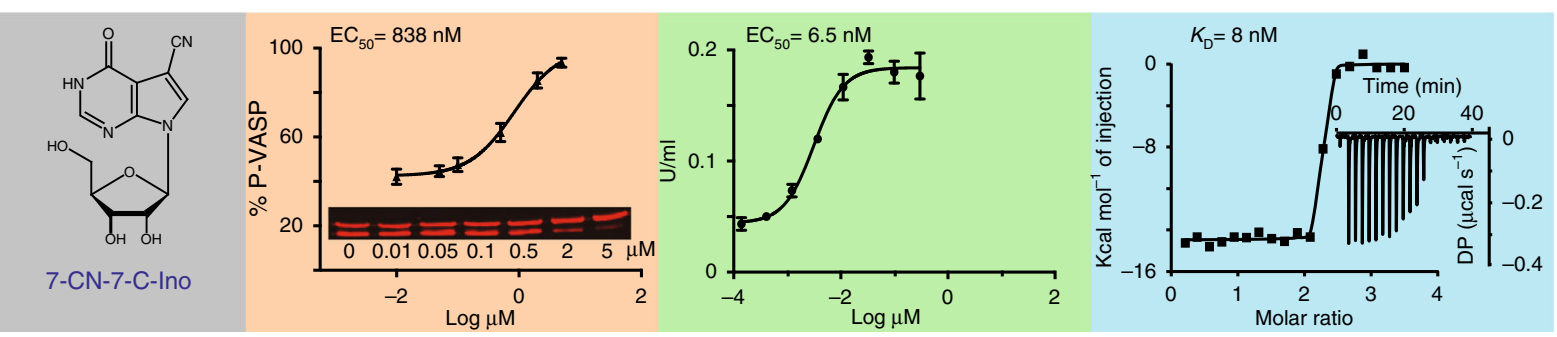

Supplementary Movie 1). The ribose moiety docks to the CNB-A site by donating two hydrogen bonds (hydroxyl groups $\mathrm{O} 3$ and O5) to E312 (Fig. 4d, Supplementary Movie 3). E310 contacts the $\mathrm{O} 2$ hydroxyl group and at the same time brings Y371 in close proximity to make a $\pi$-stacking interaction with the hypoxanthine moiety of the ligand. The free electron pair in the cyano moiety receives a hydrogen bond from the side chain of K294, while Y300 additionally shields the ligand from the solvent. In the CNB-B site (Fig. 4e, Supplementary Movie 4), E436 interacts with the $3^{\prime}$ and $5^{\prime}$ hydroxyl groups of the ribose ring. Y483 acts as a hydrophobic cap that seems to be positioned via a watermediated (w07) hydrogen bonding network formed by the main chain carbonyl group of $\mathrm{H} 431$, the side chain of $\mathrm{E} 434$ and the $\mathrm{O} 2$ hydroxyl group from the ribose. The keto group at position 6 of 
Fig. 3 Activators of trypanosome PKA. a Hit compounds with their $\mathrm{EC}_{50}$ of in vivo PKA activity (VASP reporter assay, mean \pm SD, $n=3$ independent replicates, representative western blots as inset) and their $\mathrm{EC}_{50}$ of in vitro kinase activity (kemptide phosphorylation by $T$. brucei PKAR-PKAC1 holoenzyme expressed in L. tarentolae). A representative dose response for Toyo $(n=5), 5-I-T u(n=4), 5-\operatorname{Br}-\mathrm{Tu}(n=2)$, tubercidin $(n=2)$, and sangivamycin $(n=1)$ is shown with SD of technical duplicates or triplicates. Binding parameters to T. brucei PKAR(199-499) expressed in E. coli were determined by isothermal titration calorimetry (ITC). The power differential (DP) between the reference and sample cells upon injection was measured as a function of time (inset). The main plot presents the total heat exchange per mole of injectant (integrated peak areas from inset) as a function of the molar ratio of ligand to protein. One out of three independent replicates is shown. $\mathbf{b}$ Data for 7-CN-7-C-Ino as in $\mathbf{a}$; for number of independent replicates see Table 1. The EC $C_{50}$ and $K_{D}$ values given in $\mathbf{a}$ and $\mathbf{b}$ are rounded values from Table 1. $\mathbf{c}$ Thermodynamic signature ( $\Delta G$ in blue, $\Delta H$ in green, and $-T \Delta S$ in red) compiled from ITC measurements (mean \pm SD of $n=3$ independent replicates) in $\mathbf{a}$ and $\mathbf{b}$. Source data are provided as a Source Data file

the purine ring accepts a hydrogen bond from the main chain nitrogen atom of Y486, while the secondary amine in position 1 acts as a donor to the carbonyl of K482 (Fig. 4e, Supplementary Movie 4). In the CNB-B site, the cyano group does not engage in hydrophilic interactions but instead is sandwiched between the side chains of Y485 and Y486. A sequence alignment of kinetoplastid and mammalian PKAR (Fig. 4f, g) shows that alanine residues in both pockets (A202 and A326) of bovine PKAR are substituted by glutamates in the kinetoplastid PKAR orthologues. The positively charged arginine residues responsible for neutralizing the cyclic phosphate of cAMP (R209 and R333 in bovine PKAR) are substituted in kinetoplastid PKAs by neutral amino acids with shorter side chains (V, T, A, or N). Thus, few substitutions in a highly conserved signalling protein are hallmarks of a ligand selectivity switch from cAMP to 7-CN-7C-Ino.

Molecular docking of 7-deaza nucleosides. For the series of 7deaza nucleoside analogues, we found a good correlation between computational docking to the TcPKAR structure and the potency of activation of the purified kinase (Supplementary Fig. 7 and Table 1). Re-docking of the co-crystallized compound 7-CN-7-CIno to the CNB-A and -B sites of TcPKAR gave RMSD values of 0.266 and 0.252 , respectively, for the best poses. Using the Glide E-model (GE) scoring system, the best docking poses of the compounds were ranked for the A site (7-CN-7-C-Ino > Toyo > $5-\mathrm{Br}-\mathrm{Tu}>5-\mathrm{I}-\mathrm{Tu}>$ sangivamycin $>\mathrm{Tu})$ and for the $\mathrm{B}$ site $(7-\mathrm{CN}-$ 7-C-Ino $>\mathrm{Tu}>$ Toyo $>5$ - Br- $\mathrm{Tu}>5$-I- $\mathrm{Tu}>$ sangivamycin). Interestingly, Tu docks very well in the $\mathrm{B}$ site but is a weak activator (see Discussion). The cyano-, iodo-, or bromo- groups at position 5 of all other tubercidin analogues are accommodated in a hydrophobic pocket formed by the side chains of V444, V489, Y485, and Y486, the latter three being part of the aD helix (Supplementary Fig. 7c). 7-CN-7-C-Ino forms two additional hydrogen bonds (K482/O and $\mathrm{Y} 486 / \mathrm{N}$ ) with the $\mathrm{\alpha D}$ helix compared to Toyo, correlated with a 7-fold higher affinity and 36-fold higher activation potency (Table 1).

PKA signalling and targets in trypanosomes. PKA downstream signalling components and targets are so far completely unexplored in trypanosomes. To probe PKA target phosphorylation events, we first used a phospho-specific PKA substrate antibody detecting the phosphorylated consensus PKA sites $\mathrm{RXXS}^{*} / \mathrm{T}^{*}$. A 3-4-fold increase in global $\mathrm{RXXS}^{*} / \mathrm{T}^{*}$ site phosphorylation was observed by western blotting after $10 \mathrm{~min}$ of Toyo or 7-CN-7-CIno treatment in wild type but not in $\Delta p k a r / \Delta p k a r$ cells (Fig. 5a). Phosphoproteome analysis under these conditions $(15 \mathrm{~min} \pm 7$ CN-7-C-Ino) showed 642 significantly (FDR $\leq 0.05, s_{0}=2$ ) upregulated phosphosites, mostly containing PKA motifs (77\%) (K/R-X-X-S/T, K/R-X-S/T) ${ }^{5}$, whereas these PKA motifs were underrepresented (19\%) in the 84 downregulated phosphosites (Fig. 5b, c; Supplementary Data 1). The frequency distribution of PKA motif subsets in the 7-CN-7-C-Ino-induced T. brucei phosphoproteome matches closely with that observed for human PKA motifs (Fig. 5c). An unbiased motif discovery algorithm confirmed enrichment of the same PKA motifs among the upregulated phosphosites (Supplementary Fig. 8a, b). Gene ontology (GO) enrichment analysis predicts functions of PKA in posttranscriptional regulation of gene expression, dynamics of cytoskeletal and organellar structures, signalling, and cell division and cytokinesis (Supplementary Fig. 8c).

We also explored changes in cellular protein abundance following PKA activation at later time points ( 6 and $12 \mathrm{~h}$ ) by label-free quantitative proteomics. No significantly regulated proteins were detected $6 \mathrm{~h}$ post induction and only 14 proteins $12 \mathrm{~h}$ post induction in wild type cells $(\geq 1.5$-fold difference at $p \leq$ 0.05, Supplementary Fig. 9), including PKAC1/2 and metacaspase $4(\mathrm{MCA} 4)^{53}$. The $\Delta p k a r / \Delta p k a r$ mutant trypanosomes served as negative control. As expected, PKAC1/2 levels decreased rapidly, resulting from ligand-induced dissociation of the holoenzyme complex and instability of the free $\mathrm{C}$ subunit, as reported for mammalian $\mathrm{PKA}^{37}$. MCA4, for which an antibody was available, informed on differential effects of 7-CN-7-C-Ino and Toyo: whereas both inducers decreased MCA4 abundance in wild type but not in $\Delta p k a r / \Delta p k a r$ cells $12 \mathrm{~h}$ post induction, only Toyo elevated MCA4 at later time points in a PKA-independent fashion (Fig. 5d). This is compatible with observed PKAindependent effects of Toyo on growth (Table 1) due to multiple cellular targets. 7-CN-7-C-Ino, on the other hand, does not produce these PKA-independent effects on growth, viability, or visible phenotype. This compound is therefore proposed as novel and specific tool to study in vivo the essential processes regulated by PKA in trypanosomes.

\section{Discussion}

Functionally and biochemically uncharacterized PKAs are regularly annotated as cyclic AMP-dependent protein kinases due to high conservation in the eukaryotic kingdom. Here, we have identified novel potent activators of cAMP-independent trypanosome PKA and unambiguously show that ligand selectivity has evolved away from cAMP. This challenges the current view that all PKA orthologues are cAMP-dependent and explains why earlier attempts to detect cAMP-stimulated kinase activity ${ }^{23,24}$ or binding of cAMP to recombinantly expressed domain fragments of trypanosomal PKAR orthologs 22,27 remained negative. The CNB domain seems to be a more versatile ligand-binding domain, not limited to cyclic nucleotides, as reported for some distant CNB family members in prokaryotes ${ }^{3,4}$. We expect that systematic surveys will identify novel eukaryotic CNB domain binding specificities, not only in phylogenetically distant trypanosomatids.

Several conserved amino acids in the CNB domains of PKAR from T. brucei and related kinetoplastids depart from the consensus (see Fig. 4f, g), as noted earlier $22,27,31$. Yet, the amino acid sequence alone did not allow the prediction of altered ligand specificity. For example, the consensus arginine interacting with the exocyclic phosphate of cAMP in almost all PKAs (R333 in 
a

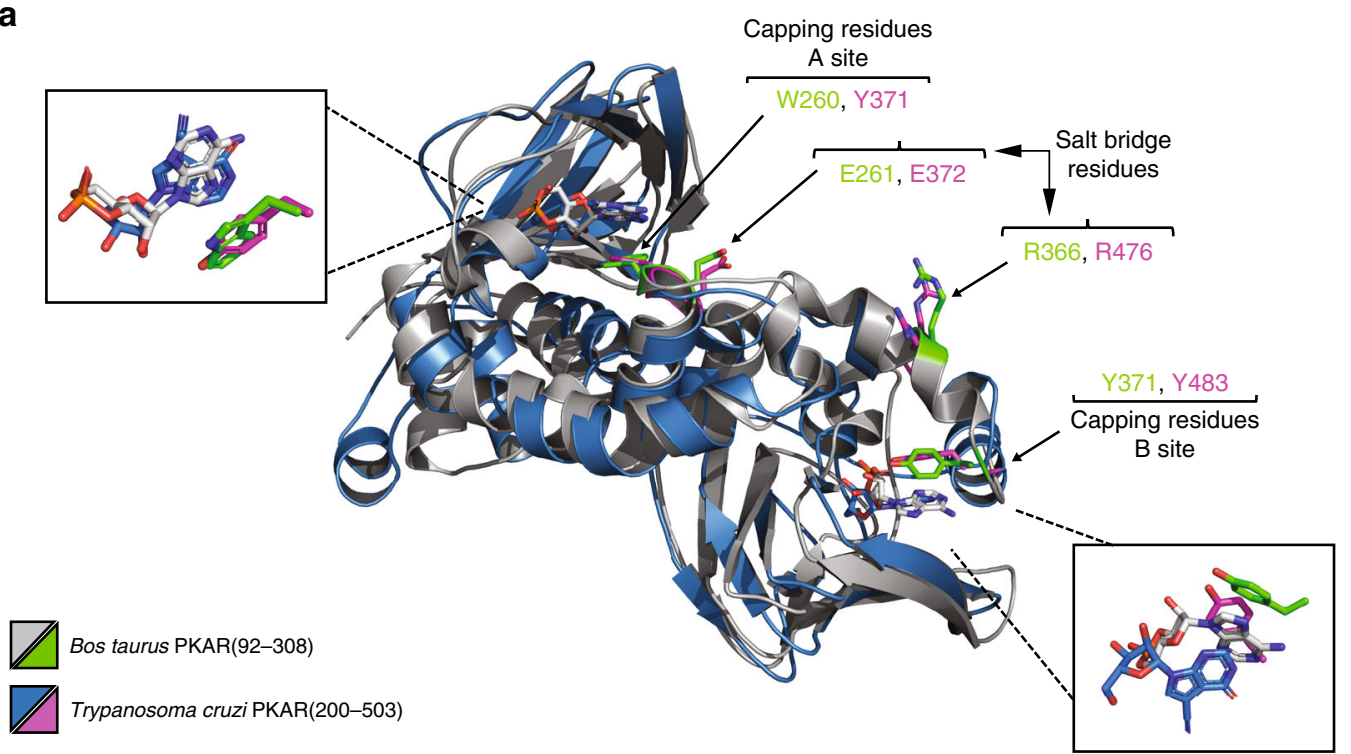

b

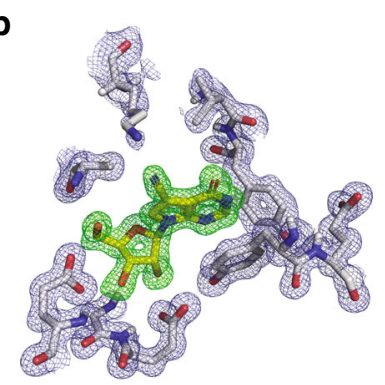

c

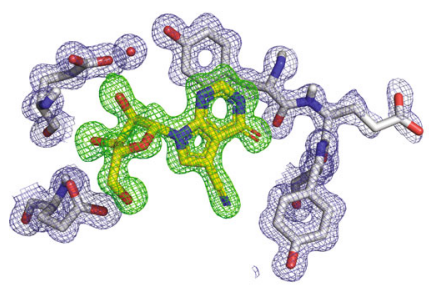

d

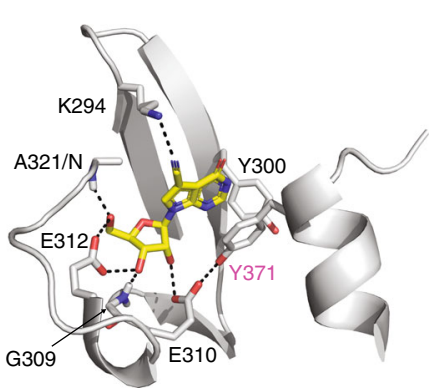

e

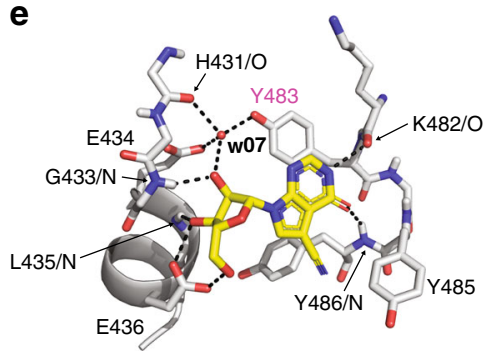

f

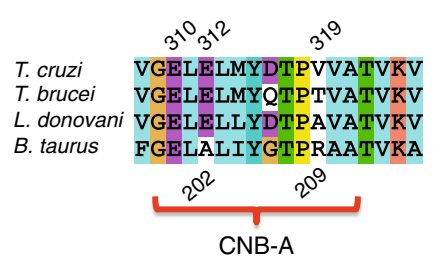

g

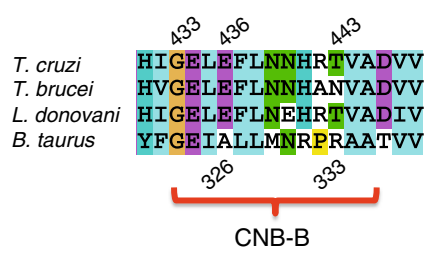

Fig. 4 Co-crystal structure of trypanosome PKAR with 7-CN-7-C-Ino. a Structural alignment of T. cruzi PKAR(200-503) (chain representation in blue) and Bos taurus PKARI $\alpha$ (92-308) (PDB 1RGS, chain representation in grey). The two capping residues and the salt bridge pair are highlighted by their carbon atoms colour-coded as green and magenta in the B. taurus and T. cruzi PKAR structures, respectively. The overlay of ligand poses is shown in the blow-ups and the $\pi$-stacking interactions in both sites for both proteins are highlighted. b, c Fo-Fc ( $3 \sigma$, green) and 2Fo-Fc (1 $\sigma$, blue) maps of TcPKAR CNB-A and CNB-B, respectively, showing 7-CN-7-C-Ino modelled to fit the electron densities. d, e Hydrogen bonding network (black dashed lines) of 7-CN-7-C-Ino bound to TCPKAR(200-503) CNB-A and CNB-B, respectively. The capping residues in CNB-A (Y371) and CNB-B (Y483) are labelled in magenta. 3D versions of $\mathbf{a}$, $\mathbf{d}$, and $\mathbf{e}$ are available as Supplementary Movie 1, 3, and 4, respectively. $\mathbf{f}$, g Sequence alignment of PKAR in CNB-A and CNB-B, respectively, of representative kinetoplastid parasites with a mammalian PKAR (B. taurus PKARI $\alpha$ ) as reference. Numbering refers to $T$. cruzi (top) and $B$. taurus (bottom), respectively

bovine RIa) is one of the key replacements in kinetoplastid PKAR, but the mammalian RIa mutant of that residue retained cAMP activation at an only 5 -fold decreased $\mathrm{EC}_{50} 54$. Our cocrystal structure shows that glutamate residues (E312/E436) present in both $\mathrm{CNB}$ pockets of TcPKAR may clash with the negatively charged phosphate of cAMP (Supplementary Movie 5) but strengthen the interaction with the ribose moiety of 7-CN-7C-Ino. In addition, an important role of the $\mathrm{aD}$ helix, an extra helix only present at the C-terminus of trypanosome PKAR is suggested. These features are conserved in the Trypanosoma species $T$. brucei and T. cruzi that share the unusual ligand binding. The high affinity of the 7-deaza nucleoside analogues can be explained by interactions with side groups on the purine ring. For example, a hydrogen bond donor-acceptor pair (Y486/ $\mathrm{N}-\mathrm{K} 482 / \mathrm{O}$ ) from the backbone of the trypanosome-specific $\mathrm{aD}$ helix (see Fig. 4e) favours the interaction with a ligand having an acceptor-donor pair at positions 1 and 6 of the purine ring. This is the case for the hypoxanthine-like purine ring in 7-CN-7-C-Ino (Fig. 4e, Supplementary Fig. 7b) but not the adenine-like purine ring in toyocamycin. In silico docking shows that the bulky cyano-, iodo-, or bromo- side groups fill a small hydrophobic pocket formed at the interface between the beta barrel (V444) and the $a \mathrm{D}$ helix (V489, Y485, Y486) in CNB-B, and correctly predicts the relative affinities of these analogues (Supplementary 

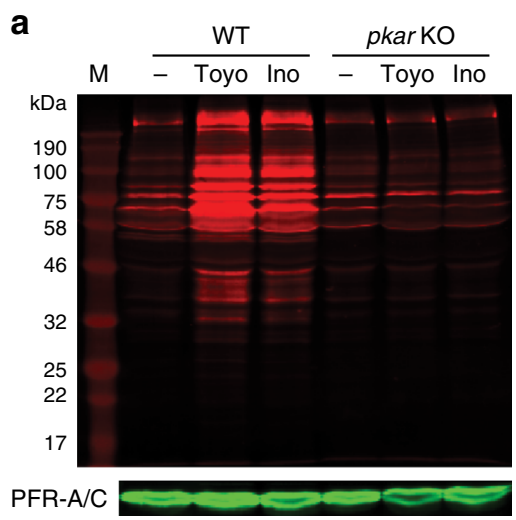

PFR-A/C

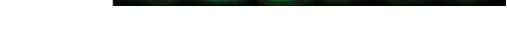

b

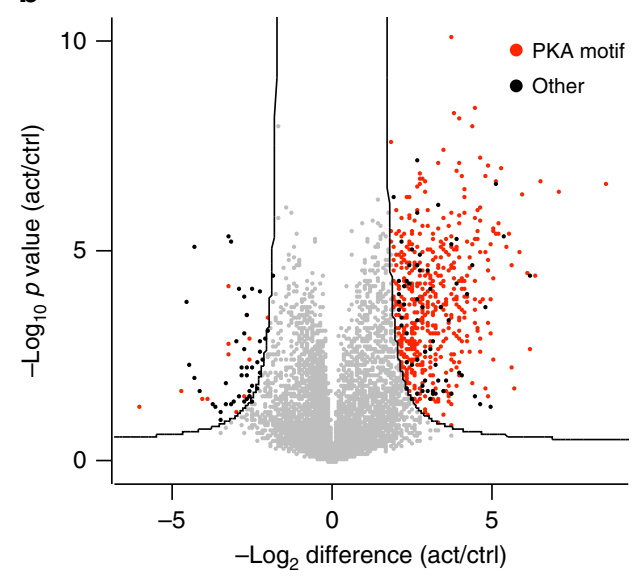

C

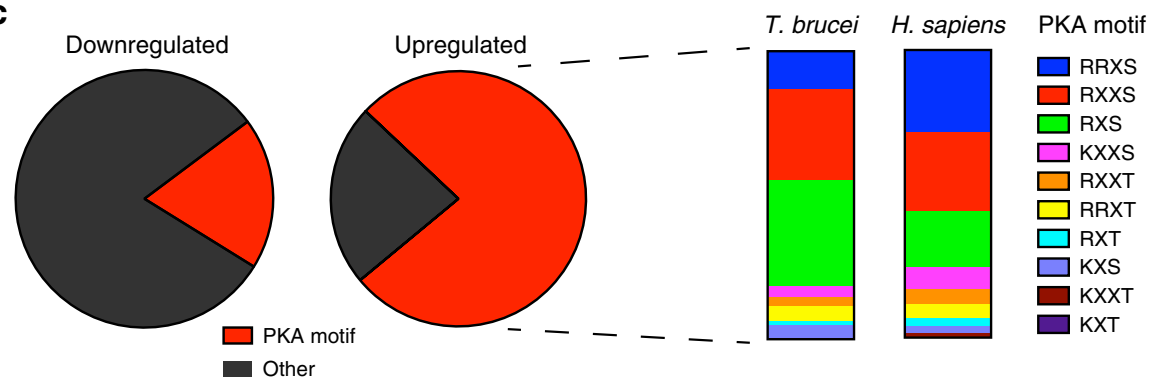

d WT

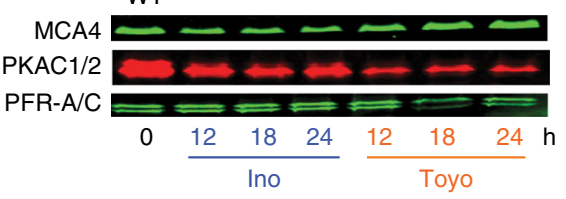

e
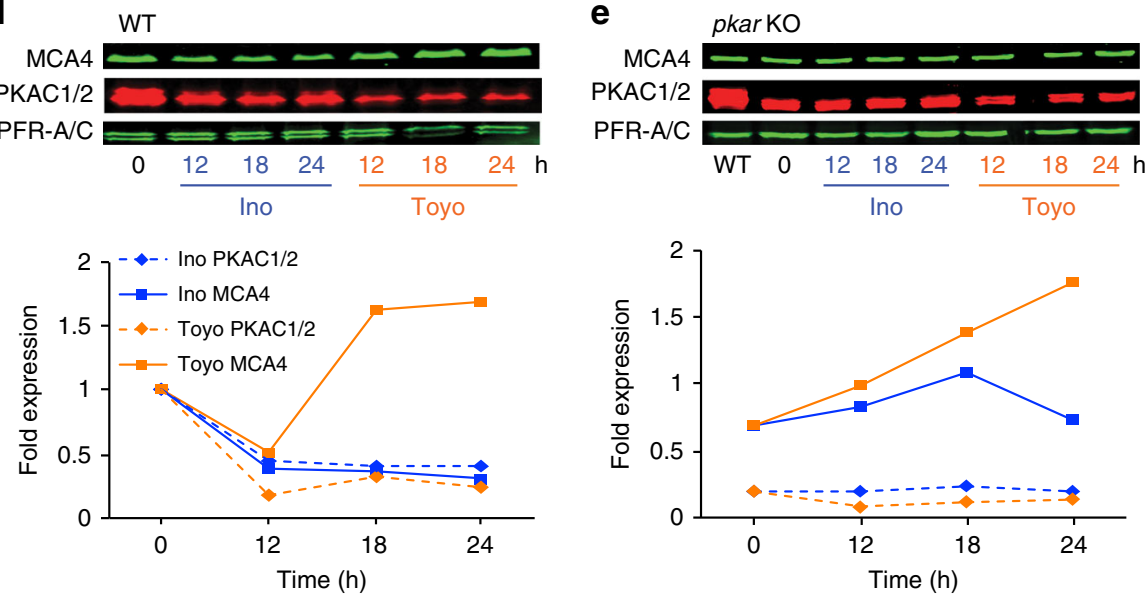

Fig. 5 Target phosphorylation and expression changes. a Global display of proteins phosphorylated at RXXS/T sites. Wild type (WT) or $\Delta p k a r / \Delta p k a r$ ( $p k a r$ KO) cells were treated or not (-) with $2 \mu \mathrm{M}$ Toyo or 7-CN-7-C-Ino (Ino) for $10 \mathrm{~min}$ and lysates were subjected to western blotting with anti-phospho-RXXS $/ \mathrm{T}^{\star}$ and anti-PFR-A/C as loading control. M: protein molecular weight marker. b Volcano plot representation of phosphopeptides quantified by label-free phosphoproteome analysis. Phosphopeptides are plotted according to $p$-value and fold change caused by treatment of $T$. brucei WT cells with 7-CN-7-C-Ino ( 8 $\mu \mathrm{M}, 15 \mathrm{~min}, n=4$ independent experiments) in comparison to untreated cells ( $n=4$ independent experiments). Phosphopeptides that change significantly in abundance (FDR $\leq 0.05, s_{0}=2$ ) and contain phosphosites matching PKA consensus motifs (R/K-X-X-S/T, R/K-X-S/T) 5 are shown as red dots; significantly changed phosphopeptides without PKA consensus motifs are shown in black. $\mathbf{c}$ Pie charts showing the fraction of PKA consensus motifs (red) within the downregulated ( $n=84$, left) or upregulated ( $n=642$, right) phosphosites. The frequency of individual subsets of PKA motifs (upregulated) was compared to the human PKA site motif frequency retrieved from the PhosphoSitePlus database (https://www.phosphosite.org). d Abundance changes of metacaspase 4 (MCA4) and PKAC1/2 in WT or e pkar KO cells after treatment with $4 \mu \mathrm{M}$ 7-CN-7-C-Ino (Ino) or Toyo in a 24-h time course. Western blot signals were normalized to the loading control PFR-A/C; untreated WT cells were set to 1. Source data to $\mathbf{a}$ and $\mathbf{c}-\mathbf{e}$ are provided as a Source Data file

Fig. 7c). The low potency of sangivamycin can also be explained by the incompatibility of its bulky and hydrophilic side group with the small hydrophobic pocket. Interestingly, the second highest affinity to the B-site is predicted for tubercidin, while the kinase activation potency of this ligand is second lowest. Tubercidin has no side group at position 7; its ribose ring can sample more conformations and therefore possibly score higher in docking. The missing contact to the $\mathrm{aD}$ helix is however correlated with low potency. This suggests a role of this trypanosomespecific helix not only for binding but also for the conformational change that underlays the activation mechanism. Only recently, the critical residues for cyclic nucleotide selectivity (cAMP/ cGMP) between PKA and PKG have been defined ${ }^{52,55}$. Mutations G316R/A336T in CNB-B convert hRIa from low to high cGMP 
affinity. Of note, the residue corresponding to A336 in hRIa is V444 in TcPKAR and is part of the hydrophobic pocket formed between beta barrel and $\mathrm{\alpha D}$ helix. This pocket accommodates the substituent at the $\mathrm{C} 7$ position of our activators and may determine their selectivity.

The structural alignment between TcPKAR and bovine PKARIa shows conservation of the general polypeptide chain folding and of the putative salt bridge and capping residues linked to the extensively studied allosteric activation mechanism in mammalian PKARIa ${ }^{1,30}$. The rapid decrease of PKAC1 levels upon treatment of cells with 7-CN-7-C-Ino (Fig. 5d, Supplementary Fig. 9a) indicates that kinase activation involves holoenzyme dissociation in vivo. The paradigmatic features of the signalling protein PKA seem to be conserved and adapted to different activating ligands by minor substitutions in the PBC in concert with the trypanosome-specific $\alpha \mathrm{D}$ helix extension in CNB-B. It follows that some of the "orphan" CNB domains identified in eukaryotic genomes $2,31,56,57$ may be regulated by unexpected and novel ligands. Such discoveries will be as insightful as was the discovery of ligands for other classes of orphan receptors, e.g. from the G-protein coupled receptor ${ }^{58}$ or nuclear hormone receptor ${ }^{59}$ families.

Our report on this unique cAMP-independent PKA may appear to question the role of cAMP as second messenger in trypanosomes. Homologs of other known effector proteins like Epac or cyclic nucleotide gated ion channels were not found in the T. brucei genome. Yet, cAMP and the enzymes for its production and degradation are present and play vital roles in cell division and host immunity subversion ${ }^{12,17-20}$. We have previously identified a number of potential cAMP response proteins (CARPs) by genome-wide RNAi library screening in T. brucei ${ }^{29}$. CARP1 has three CNB domains and the T. cruzi ortholog ${ }^{60}$ binds cAMP. Interestingly, CARP1 is exclusively found in kinetoplastid genomes and possibly coevolved with the ligand swap of PKA away from CAMP. There are also additional candidate genes with predicted CNB domains, some of which may be cAMP effectors $^{41,60}$. Divergent CNB domains as found in cAMPbinding Popeye-domain proteins ${ }^{61}$ might be present but not recognized due to limited homology. Together, the cAMPindependence of PKA in trypanosomes is well compatible with cAMP signalling in these organisms.

7-CN-7-C-Ino is a potent activator and has low toxicity in vivo, in contrast to the 7-deazaadenosine analogues. The latter compounds are known to have pleiotropic effects in various cell types by interfering with cellular processes involving nucleosides or nucleotides. This comprises inhibition of kinases and inhibition of DNA, RNA, and protein synthesis (reviewed in ref. ${ }^{47}$ ) and explains the broad antibiotic and antineoplastic activity profile. A possible reason for the reduced off-target effects of 7-CN-7-C-Ino might be lower in vivo stability of the drug. This should not affect the short term in vivo kinase reporter assay but reduce off-target effects on growth and viability. In trypanosomes, toxicity of the 7deazaadenosine analogues is clearly not mediated by PKA, as we find no difference between WT and $\Delta p k a r / \Delta p k a r$ cells. A base level of catalytic PKA function is essential for growth and viability of T. brucei (Supplementary Fig. 2). Therefore, efficient inhibition of PKA activation by trapping the catalytic subunits in inactive holoenzyme complexes would reduce the free kinase to lethal level. The challenge in view of possible antiparasitic drug development will be to turn our novel activators to PKA inhibitors similar to what had been achieved for mammalian $\mathrm{PKA}^{62}$. As this work shows, TbPKA is essential, druggable, and has a ligand specificity differing from PKAR of the mammalian host. Due to conservation of the substitutions in the CNBs in different kinetoplastid PKAs, such inhibitors may also target the orthologues of related kinetoplastid pathogens like Leishmania.
With 7-CN-7-C-Ino, we contribute a potent chemical tool that will spur further investigations on the cellular PKA targets and physiological functions in trypanosomes. Our 7-CN-7-C-Inoinduced phosphoproteome returned a surprising number of highly upregulated P-sites, mostly (77\%) within PKA consensus motifs. The GO terms most enriched among the target proteins are related to posttranscriptional control of gene expression and signalling. PKA phosphorylates directly or indirectly 17 other kinases, indicating interconnection with other signalling pathways. Participation in posttranscriptional control is not surprising as many RNA binding proteins and regulators of RNA stability or translation are regulated by phosphorylation in many organisms ${ }^{63}$. In trypanosomes, gene expression is almost exclusively controlled at the posttranscriptional level ${ }^{64}$. Therefore, PKA that participates in transcriptional regulation in other eukaryotes, may be redirected to the targets that exert gene expression control in trypanosomes. The cytokinesis phenotypes observed upon genetic perturbation of TbPKA (Supplementary Fig. 2 and ref. ${ }^{35}$ ) correspond well to enriched GO terms related to cell division and cytokinesis. The enrichment for cytoskeletal structures and the flagellum perfectly correspond to the subcellular localization of PKAR in the flagellum ${ }^{65}$, a motility phenotype ${ }^{65}$ and cytokinesis, a process that depends on the flagellum ${ }^{66}$. Surprisingly, the quantitative proteome analysis upon induction with 7-CN-7-CIno returned only 14 proteins significantly regulated in abundance after $12 \mathrm{~h}$. Among them, downregulation of MCA4, an unconventional metacaspase regarded as pseudopeptidase ${ }^{53}$ is interesting, since T. brucei mutants with homozygous deletion of MCA4 have reduced virulence in animal infections ${ }^{53}$.

The in vivo pathways and mechanisms upstream of PKA in trypanosomes are completely elusive to date. Is trypanosome PKA the effector of an unknown alternative second messenger? The direct activators of T. brucei PKA identified by this work include tubercidin, toyocamycin, and sangivamycin, natural antibiotics that are secondary metabolites of Streptomyces strains ${ }^{67}$. However, it seems unlikely that 7 -deazapurines are the endogenous PKA ligands, since no homologs of the Streptomyces genes encoding enzymes required for synthesis of the precursor preQ0 ${ }^{68}$ were detected in the T. brucei genome. Of course, an alternative route of synthesis of a 7-deazapurine cannot be excluded. As we find indirect activation of T. brucei PKA by dipyridamole in live cells, this antimetabolite may perturb the parasite's nucleoside metabolism, e.g. by blocking uptake of adenosine ${ }^{69}$. Nucleoside-related metabolites or by-products of purine salvage may have adopted the role of second messengerlike molecules targeting PKA in trypanosomes. The identity of the physiological PKA ligand and the respective pathway is an exciting line of current research.

\section{Methods}

Trypanosome culture conditions. Bloodstream forms of the monomorphic Try panosoma brucei brucei strain Lister 427 , variant MiTat $1.2^{70}$, were cultivated at 37 ${ }^{\circ} \mathrm{C}$ and $5 \% \mathrm{CO}_{2}$ in modified HMI-9 medium ${ }^{14}$ supplemented with $10 \%(\mathrm{v} / \mathrm{v}$ ) heat inactivated fetal bovine serum (FBS). Cell lines $13-90^{71}$ or $1313-514^{72}$ expressing T7 polymerase and Tet repressor were kept under continuous selection with $2.5 \mu \mathrm{g}$ $\mathrm{ml}^{-1} \mathrm{G} 418$ and $5 \mu \mathrm{g} \mathrm{ml}^{-1}$ hygromycin B or $0.2 \mu \mathrm{g} \mathrm{ml}^{-1}$ phleomycin and $2 \mu \mathrm{g} \mathrm{ml}^{-1}$ G418, respectively.

Immunopurifications. PTP purification was performed according to the protocol of Schimanski et al. ${ }^{73}$ with a few modifications. Briefly, trypanosomes expressing PTPtagged PKAR from the endogenous locus were lysed in PA-150 buffer (w/o DTT, supplemented with Complete Mini EDTA-free protease inhibitor cocktail (Roche) and $25 \mu \mathrm{g} \mathrm{ml}^{-1}$ pepstatin A) by three sonication cycles $30 \mathrm{~s}$ each with the Bioruptor device (Diagenode) at low power. After centrifugation $\left(20 \mathrm{~min}, 20,000 \times \mathrm{g}, 4^{\circ} \mathrm{C}\right)$, the cleared supernatant was incubated with IgG beads (pre-equilibrated with PA-150 buffer) for $4 \mathrm{~h}$ to overnight by overhead rotation at low speed. Two washes with PA150 buffer and one with PBS were followed by elution of proteins bound to the IgG beads by incubation with $2 \times$ Laemmli sample buffer ( $125 \mathrm{mM}$ Tris, $\mathrm{pH} 6.8,4 \%$ (w/v) SDS, $20 \%(\mathrm{v} / \mathrm{v})$ glycerol, $10 \%$ 2-mercaptoethanol, $0.02 \%(\mathrm{w} / \mathrm{v})$ bromophenol blue) for 
5 min at $95^{\circ} \mathrm{C}$. Immunoprecipitation of Tyl- or HA-tagged PKA subunits was performed by incubation of trypanosomes in lysis buffer $(50 \mathrm{mM}$ Tris, $\mathrm{pH} 7.2,2 \mathrm{mM}$ EGTA, $150 \mathrm{mM} \mathrm{NaCl}, 0.2 \% \mathrm{NP}-40,1 \mathrm{mM} \mathrm{NaVO}_{4}, 0.5 \%$ aprotinin, $2 \mu \mathrm{g} \mathrm{ml}^{-1}$ leupeptin, $1 \mathrm{mM}$ PMSF) for $10 \mathrm{~min}$ on ice with subsequent clearing of the supernatant by centrifugation $\left(20 \mathrm{~min}, 20,000 \times g, 4^{\circ} \mathrm{C}\right)$. The cleared lysate was incubated with the respective epitope-tag antibody coupled covalently (using the cross-linker DMP (Thermo Scientific) according to the manufacturer's instructions) or non-covalently to protein G sepharose beads (Amersham Pharmacia) for $1 \mathrm{~h}$ to overnight.

In vivo PKA reporter assay. Trypanosomes were harvested $(10 \mathrm{~min}, 1400 \times g$, $37^{\circ} \mathrm{C}$ ) and resuspended in HMI-9 medium (pre-heated to $37^{\circ} \mathrm{C}$ ) to a density of $5 \times 10^{7}$ cells ml ${ }^{-1}$. After a $5-10 \mathrm{~min}$ recovery at $37^{\circ} \mathrm{C}$ with mild shaking, test compounds or solvent were added to the cell suspension followed by careful mixing. After incubation at $37^{\circ} \mathrm{C}$ for the specified time period, cells were lysed with 6x Laemmli sample buffer preheated to $95^{\circ} \mathrm{C}$ and incubated for $5 \mathrm{~min}$ at $95^{\circ} \mathrm{C}$. Incubation of trypanosomes at this density for up to $30 \mathrm{~min}$ had no effect on VASP phosphorylation (Supplementary Fig. 3g). GraphPad Prism 7.0 was used for $\mathrm{EC}_{50}$ calculation by non-linear regression analysis using an equation for a sigmoidal dose-response curve with variable slope. The same software was used for visualization of all graphs and bar charts.

\section{Inhibitors and nucleoside analogues. PKI(5-24) and myr-PKI(14-22) were} obtained from Biomol. CpdA ${ }^{19}$ (now renamed as NPD-001) ${ }^{74}$ was synthesized by Geert-Jan Sterk, Mercachem. Dipyridamole was obtained from Sigma-Aldrich, toyocamycin and sangivamycin from Berry \& Associates. All other nucleoside and cyclic nucleotide analogues were obtained from Biolog GmbH, Bremen. 7-CN-7-CIno was synthesized according to Hinshaw et al. ${ }^{75}$ with $125 \mathrm{mg}(429.1 \mu \mathrm{mol})$ Toyo as starting material. By variation to the original protocol, the raw product was purified by reversed phase medium pressure liquid chromatography (MPLC). Briefly, the raw product was dissolved in $20 \mathrm{ml}$ water, filtrated and applied to a Merck LiChroprep ${ }^{\circ}$ RP-18 column $(15-25 \mu \mathrm{M} ; 125 \times 35 \mathrm{~mm})$, previously equilibrated with water. The column was washed with water to remove excess of inorganic salts and hydrophilic impurities. Afterwards, $1 \%$ and 2\% 2-propanol in water was used to elute the target compound. Product-containing fractions were concentrated by rotary evaporation under reduced pressure and subsequently freezedried to yield $89.34 \mathrm{mg}(305.7 \mu \mathrm{mol})$ of $7-\mathrm{CN}-7-\mathrm{C}$-Ino with a purity of $99.93 \%$ by analytical HPLC (ODS-A 120-11, RP-18 (YMC, Dinslaken, Germany); $250 \times 4$ $\mathrm{mm}$; $9 \%$ acetonitrile, $20 \mathrm{mM}$ triethylammonium buffer, $\mathrm{pH} 6.8 ; 1.0 \mathrm{ml} \mathrm{min}^{-1}$; UVdetection at $265 \mathrm{~nm}$ ).

Leishmania tarentolae expression system. The L. tarentolae strain LEXSY T7-TR (Jena Biosciences) was cultivated at $26.5^{\circ} \mathrm{C}$ in BHI medium supplemented with 10 $\mu \mathrm{g} \mathrm{ml}^{-1}$ hemin, $100 \mathrm{U} \mathrm{L}^{-1}$ streptomycin and $100 \mathrm{mg} \mathrm{L}^{-1}$ penicillin according to the protocols provided by Jena Biosciences. For maintenance of $\mathrm{T} 7$ polymerase and Tet repressor, $10 \mu \mathrm{g} \mathrm{ml}^{-1}$ nourseothricin (NTC) and $10 \mu \mathrm{g} \mathrm{ml}^{-1}$ hygromycin B were added to the medium. For co-expression of T. brucei PKAR-10 $\times$ His and StrepPKAC1, the full length ORFs were amplified from genomic DNA using primers introducing the respective epitope tag and cloned into pLEXSY_I-ble3 and pLEXSY_I-neo3 (Jena Biosciences), respectively. Details on primer sequences and cloning strategies are available upon request. Cells transfected with both constructs were cultivated in the presence of $100 \mu \mathrm{g} \mathrm{ml}^{-1}$ phleomycin and $50 \mu \mathrm{g} \mathrm{ml}^{-1} \mathrm{G} 418$. L. tarentolae cells at mid log phase $\left(2-3 \times 10^{7}\right.$ cells ml$\left.^{-1}\right)$ were induced with $10 \mu \mathrm{g}$ $\mathrm{ml}^{-1}$ tetracycline for $24 \mathrm{~h}$. Lysis of cells in $50 \mathrm{mM}$ Tris, $\mathrm{pH} 7.4,150 \mathrm{mM} \mathrm{NaCl}, 0.2 \%$ Triton X-100, 1 mM 2-mercaptoethanol was completed by a Dounce homogenizer. Tandem affinity purification of the holoenzyme complex was performed to guarantee subunit stoichiometry and highest purity: His-tag purification using Ni-NTA beads (Thermo Fisher Scientific) was followed by Strep-tag purification using gravity flow chromatography and StrepTactin sepharose beads (IBA), according to the manufacturers' instructions. The eluted fractions were pooled and dialyzed against the kinase storage buffer (20 mM MOPS, pH 7.0, $150 \mathrm{mM} \mathrm{NaCl}, 1 \mathrm{mM}$ 2-mercaptoethanol).

In vitro kinase assay. A radioactive PKA kinase assay was performed according to Hastie et al. ${ }^{76}$, using $100 \mu \mathrm{M}$ Kemptide (LRRASLG) as kinase substrate and $100 \mu \mathrm{M}$ ATP spiked with $\left[\gamma^{-32} \mathrm{P}\right]$ ATP to give $200-400 \mathrm{cpm} \mathrm{pmol}^{-1}$. GraphPad Prism 7.0 was used for $\mathrm{EC}_{50}$ calculation by non-linear regression analysis using an equation for a sigmoidal dose-response curve with variable slope. The same software was used for visualization of all graphs and bar charts.

Binding studies by isothermal titration calorimetry. N-terminally truncated $T$. brucei PKAR (aa 199-499) was cloned into pETDuet-1 (Novagene) with an Nterminal $\mathrm{His}_{6}$-tag and expressed in E. coli Rosetta (DE3). The bacteria were grown in Luria-Bertani (LB) medium to $\mathrm{OD}_{600}$ of $\sim 0.4$ at $37^{\circ} \mathrm{C}$, followed by overnight induction with $0.4 \mathrm{mM}$ IPTG at $20^{\circ} \mathrm{C}$. Cells were harvested by centrifugation and lysed in a French Press. Protein purification was done by Ni-NTA affinity chromatography, followed by elution in $50 \mathrm{mM}$ HEPES, $\mathrm{pH} 7.5,50 \mathrm{mM} \mathrm{NaCl}$, and 250 $\mathrm{mM}$ imidazole. Eluted protein $\left(\sim 7 \mathrm{mg} \mathrm{ml}^{-1}\right)$ was dialyzed overnight in $50 \mathrm{mM}$ HEPES, pH 7.5, $50 \mathrm{mM} \mathrm{NaCl}, 1 \mathrm{mM}$ DTT, and stored at $-80^{\circ} \mathrm{C}$. Ligand-free protein for binding studies was prepared by adding solid urea to the protein solution to a final concentration of $8 \mathrm{M}$. After $1 \mathrm{~h}$ at $25^{\circ} \mathrm{C}$, the solution was passed over a prepacked PD10 column (GE Healthcare) equilibrated with $8 \mathrm{M}$ urea, 50 mM HEPES, pH 8.5, $50 \mathrm{mM} \mathrm{NaCl}$. TbPKAR(199-499) was refolded overnight by dialysis against $50 \mathrm{mM}$ Tris, pH $8.5,240 \mathrm{mM} \mathrm{NaCl}, 10 \mathrm{mM} \mathrm{KCl}, 2 \mathrm{mM} \mathrm{MgCl}_{2}, 2$ $\mathrm{mM} \mathrm{CaCl}, 0.4 \mathrm{M}$ sucrose, $1 \mathrm{mM} \mathrm{DTT}$ at $4{ }^{\circ} \mathrm{C}$, followed by separation of monomer from aggregates by size-exclusion chromatography on a Superdex 200 Increase 10/ 300 GL column (GE Healthcare) equilibrated with $50 \mathrm{mM}$ HEPES, pH 7.5, $50 \mathrm{mM}$ $\mathrm{NaCl}$, and $1 \%$ DMSO (buffer A). Eluted protein was diluted to $10 \mu \mathrm{M}$ for ITC. The human PKARIa (full size) was expressed from the pETDuet-1 based plasmid $6 \mathrm{H}$. tev/HsPKARa in E. coli Rosetta D3, prepared cAMP-free according to Buechler et al. ${ }^{77}$ with the following modification: the refolding buffer was the buffer used for TbPKAR refolding. The purified protein was diluted to $5 \mu \mathrm{M}$. For binding assays, 13-19 injections of 2-3 $\mu \mathrm{l}$ were performed with a MicroCal PEAQ-ITC (Malvern) instrument. In each series, $100 \mu \mathrm{M}$ of ligand (prepared using buffer A) was injected at $298 \mathrm{~K}$ into $5-10 \mu \mathrm{M}$ protein freshly eluted from size exclusion chromatography. Binding constants and thermodynamic data were derived from best least square fit analysis, applying a model with two binding sites (performed with MicroCal PEAQ-ITC software)

Quantitative phosphoproteomics. For each sample, $6 \times 10^{8}$ T. brucei cells treated or not with $8 \mu \mathrm{M}$ 7-cyano-7-deazainosine for $15 \mathrm{~min}$ were lysed in $300 \mu \mathrm{l} 4 \%$ (w/v) sodium deoxycholate, $0.1 \mathrm{M}$ Tris, $\mathrm{pH} 8.5$ for $5 \mathrm{~min}$ at $95^{\circ} \mathrm{C}$ (according to the protocol of Humphrey et al. ${ }^{78}$ ). Samples were sonicated using a Bioruptor (Diagenode) (high power, two cycles of $10 \mathrm{~min}$ each, $30 \mathrm{~s}$ on/off). Protein concentration was determined by BCA protein assay and samples were adjusted to equal concentrations. Sample preparation and mass spectrometry were exactly carried out as described by Humphrey et al. ${ }^{78}$. MaxQuant 1.5.2.8 ${ }^{79}$ was used to identify proteins and quantify by LFQ with the following parameters: Database, TriTrypDB39 TbruceiTREU927_AnnotatedProteins; MS tol, 10 ppm; MS/MS tol, 0.5 Da; Peptide FDR, 0.1; Protein FDR, 0.01 Min. peptide Length, 5; Variable modifications, Oxidation (M), Phosphorylation (STY); Fixed modifications, Carbamidomethyl (C); Peptides for protein quantitation, razor and unique; Min. peptides, 1; Min. ratio count, 2. For proteomic analysis, identified proteins were considered as statistically significant with FDR $\leq 0.05$ and $s_{0}=1$ (two-sided Student's $T$-test adjusted for multiple comparisons by Benjamini-Hochberg correction, Perseus ${ }^{80}$ ). Phosphopeptide analysis was carried out in Perseus as suggested by Humphrey et al. ${ }^{78}$. The mass spectrometry phosphoproteomics data have been deposited to the ProteomeExchange Consortium via the PRIDE partner repository (https:// www.ebi.ac.uk/pride/archive) with the dataset identifier PXD012245. GO enrichment analysis was performed in TriTrypDB with default settings and visualized in Revigo (http://revigo.irb.hr). The motif discovery tool MoMo (using the motif-x algorithm) implemented in the MEME suite (http://meme-suite.org/) was used for unbiased motif discovery in the phosphoproteome dataset with the T. brucei TriTrypDB-40_TbruceiTREU927 protein database as background. Enriched sequence logos were visualized using CLC Main Workbench 7 (https://www. qiagenbioinformatics.com/).

Reporting summary. Further information on experimental design is available in the Nature Research Reporting Summary linked to this article.

\section{Data availability}

The coordinates of the T. cruzi PKAR crystal structure bound to 7-CN-7-C-Ino have been deposited in the Protein Data Bank under the code PDB 6FTF. The phosphoproteome and proteome datasets are available in the PRIDE partner repository with the dataset identifiers PXD012245 and PXD009073, respectively. Genome sequence and annotation information was obtained from TritrypDB (http://www.tritrypdb.org). Human PKA substrates and phosphorylation motifs were retrieved from the PhosphoSitePlus database (https://www.phosphosite.org). Gene ontology (GO) enrichment analysis was visualized using Revigo (http://revigo.irb.hr). The motif discovery tool MoMo implemented in the MEME suite (http://meme-suite.org) was used for unbiased motif discovery in the phosphoproteome dataset. The source data underlying Figs. 1c, d, 2a-h, 3a-c, 5a, c-e, Table 1, and Supplementary Figs. 1b, 2, 3a-d, $\mathrm{f}-\mathrm{i}, 4 \mathrm{a}-\mathrm{h}, 8 \mathrm{a}-\mathrm{c}, 9 \mathrm{a}-\mathrm{c}$ are provided as Source Data file.

Received: 7 July 2018 Accepted: 7 March 2019

Published online: 29 March 2019

\section{References}

1. Taylor, S. S., Ilouz, R., Zhang, P. \& Kornev, A. P. Assembly of allosteric macromolecular switches: lessons from PKA. Nat. Rev. Mol. Cell Biol. 13, 646-658 (2012)

2. Kannan, N. et al. Evolution of allostery in the cyclic nucleotide binding module. Genome Biol. 8, R264 (2007).

3. Körner, H., Sofia, H. J. \& Zumft, W. G. Phylogeny of the bacterial superfamily of Crp-Fnr transcription regulators: exploiting the metabolic spectrum by 
controlling alternative gene programs. FEMS Microbiol. Rev. 27, 559-592 (2003).

4. Joyce, M. G. et al. CprK crystal structures reveal mechanism for transcriptional control of halorespiration. J. Biol. Chem. 281, 28318-28325 (2006).

5. Shabb, J. B. Physiological substrates of cAMP-dependent protein kinase. Chem. Rev. 101, 2381-2411 (2001).

6. Scott, J. D., Dessauer, C. W. \& Tasken, K. Creating order from chaos: cellular regulation by kinase anchoring. Annu. Rev. Pharmacol. Toxicol. 53, 187-210 (2013).

7. Jia, Y. et al. Crosstalk between PKA and PKG controls pH-dependent host cell egress of Toxoplasma gondii. EMBO J. 36, 3250-3267 (2017).

8. Sugi, T. et al. Toxoplasma gondii cyclic AMP-dependent protein kinase subunit 3 is involved in the switch from tachyzoite to bradyzoite development. mBio 7, https://doi.org/10.1128/mBio.00755-16 (2016).

9. Dawn, A. et al. The central role of cAMP in regulating Plasmodium falciparum merozoite invasion of human erythrocytes. PLoS Pathog. 10, e1004520 (2014).

10. Peng, M. et al. Spatial organization in protein kinase A signaling emerged at the base of animal evolution. J. Proteome Res. 14, 2976-2987 (2015).

11. Brun, R., Blum, J., Chappuis, F. \& Burri, C. Human African trypanosomiasis. Lancet 375, 148-159 (2010).

12. Makin, L. \& Gluenz, E. cAMP signalling in trypanosomatids: role in pathogenesis and as a drug target. Trends Parasitol. 31, 373-379 (2015).

13. Saldivia, M., Ceballos-Perez, G., Bart, J. M. \& Navarro, M. The AMPKalpha1 pathway positively regulates the developmental transition from proliferation to quiescence in Trypanosoma brucei. Cell Rep. 17, 660-670 (2016).

14. Vassella, E., Reuner, B., Yutzy, B. \& Boshart, M. Differentiation of African trypanosomes is controlled by a density sensing mechanism which signals cell cycle arrest via the cAMP pathway. J. Cell Sci. 110, 2661-2671 (1997).

15. Laxman, S., Riechers, A., Sadilek, M., Schwede, F. \& Beavo, J. A. Hydrolysis products of cAMP analogs cause transformation of Trypanosoma brucei from slender to stumpy-like forms. Proc. Natl Acad. Sci. USA 103, 19194-19199 (2006).

16. Mony, B. M. et al. Genome-wide dissection of the quorum sensing signalling pathway in Trypanosoma brucei. Nature 505, 681-685 (2014).

17. Salmon, D. et al. Adenylate cyclases of Trypanosoma brucei inhibit the innate immune response of the host. Science 337, 463-466 (2012).

18. Salmon, D. et al. Cytokinesis of Trypanosoma brucei bloodstream forms depends on expression of adenylyl cyclases of the ESAG4 or ESAG4-like subfamily. Mol. Microbiol. 84, 225-242 (2012).

19. de Koning, H. P. et al. Pharmacological validation of Trypanosoma brucei phosphodiesterases as novel drug targets. J. Infect. Dis. 206, 229-237 (2012).

20. Oberholzer, M. et al. The Trypanosoma brucei cAMP phosphodiesterases TbrPDEB1 and TbrPDEB2: flagellar enzymes that are essential for parasite virulence. FASEB J. 21, 720-731 (2007).

21. Oberholzer, M., Saada, E. A. \& Hill, K. L. Cyclic AMP regulates social behavior in African trypanosomes. mBio 6, https://doi.org/10.1128/mBio.01954-14 (2015).

22. Shalaby, T., Liniger, M. \& Seebeck, T. The regulatory subunit of a cGMPregulated protein kinase A of Trypanosoma brucei. Eur. J. Biochem. 268, 6197-6206 (2001).

23. Walter, R. D. Adenosine $3^{\prime}, 5^{\prime}$-cyclic monophosphate-binding proteins from Trypanosoma gambiense. Hoppe-Seylers Z. Physiol. Chem. 359, 607-612 (1978).

24. Walter, R. D. Multiple protein kinases from Trypanosoma gambiense. HoppeSeylers Z. Physiol. Chem. 359, 601 (1978).

25. Boshart, M. \& Mottram, J. C. in Trypanosomiasis and Leishmaniasis: Biology and Control (eds. Hide, G. et al.) 227-244 (CAB International, Wallingford, 1997).

26. Bachmaier, S. \& Boshart, M. in Protein Phosphorylation in Parasites. Vol. 5 Drug Discovery in Infectious Diseases (eds. Doerig, C. et al.) Ch. 5, 99-122 (Wiley-VHC Verlag GmbH \& Co. KGaA, Weilheim, 2014).

27. Bubis, J. et al. The gene product of a Trypanosoma equiperdum ortholog of the cAMP-dependent protein kinase regulatory subunit is a monomeric protein that is not capable of binding cyclic nucleotides. Biochimie 146, 166-180 (2018).

28. Kramer, S., Klockner, T., Selmayr, M. \& Boshart, M. Interstrain sequence comparison, transcript map and clonal genomic rearrangement of a $28 \mathrm{~kb}$ locus on chromosome 9 of Trypanosoma brucei. Mol. Biochem. Parasitol. 151, 129-132 (2007).

29. Gould, M. K. et al. Cyclic AMP effectors in African trypanosomes revealed by genome-scale RNA interference library screening for resistance to the phosphodiesterase inhibitor CpdA. Antimicrob. Agents Chemother. 57, 4882-4893 (2013).

30. Kim, C., Cheng, C. Y., Saldanha, S. A. \& Taylor, S. S. PKA-I holoenzyme structure reveals a mechanism for cAMP-dependent activation. Cell 130, 1032-1043 (2007).
31. Mohanty, S. et al. Structural and evolutionary divergence of cyclic nucleotide binding domains in eukaryotic pathogens: implications for drug design. Biochim. Biophys. Acta 1854, 1575-1585 (2015).

32. Parsons, M., Worthey, E. A., Ward, P. N. \& Mottram, J. C. Comparative analysis of the kinomes of three pathogenic trypanosomatids: Leishmania major, Trypanosoma brucei and Trypanosoma cruzi. BMC Genomics 6, 127 (2005).

33. Hochstrasser, M. \& Nelson, D. L. Cyclic AMP-dependent protein kinase in Paramecium tetraurelia. Its purification and the production of monoclonal antibodies against both subunits. J. Biol. Chem. 264, 14510-14518 (1989).

34. Mutzel, R., Lacombe, M. L., Simon, M. N., de Gunzburg, J. \& Veron, M. Cloning and cDNA sequence of the regulatory subunit of cAMP-dependent protein kinase from Dictyostelium discoideum. Proc. Natl Acad. Sci. USA 84, 6-10 (1987).

35. Jones, N. G. et al. Regulators of Trypanosoma brucei cell cycle progression and differentiation identified using a kinome-wide RNAi screen. PLoS Pathog. 10 e1003886 (2014).

36. Hammarton, T. C., Monnerat, S. \& Mottram, J. C. Cytokinesis in trypanosomatids. Curr. Opin. Microbiol. 10, 520-527 (2007).

37. Amieux, P. S. et al. Compensatory regulation of RIalpha protein levels in protein kinase A mutant mice. J. Biol. Chem. 272, 3993-3998 (1997).

38. Bachmaier, S. et al. Protein kinase A signaling during bidirectional axenic differentiation in Leishmania. Int. J. Parasitol. 46, 75-82 (2016).

39. Cheek, S., Zhang, H. \& Grishin, N. V. Sequence and structure classification of kinases. J. Mol. Biol. 320, 855-881 (2002).

40. Glass, D. B., Cheng, H. C., Mende-Mueller, L., Reed, J. \& Walsh, D. A. Primary structural determinants essential for potent inhibition of cAMPdependent protein kinase by inhibitory peptides corresponding to the active portion of the heat-stable inhibitor protein. J. Biol. Chem. 264, 8802-8810 (1989).

41. Laxman, S. \& Beavo, J. A. Cyclic nucleotide signaling mechanisms in trypanosomes: possible targets for therapeutic agents. Mol. Interv. 7, 203-215 (2007).

42. Haffner, C. et al. Molecular cloning, structural analysis and functional expression of the proline-rich focal adhesion and microfilament-associated protein VASP. EMBO J. 14, 19-27 (1995).

43. Schultz, C. et al. Acetoxymethyl esters of phosphates, enhancement of the permeability and potency of cAMP. J. Biol. Chem. 268, 6316-6322 (1993).

44. Rondina, M. T. \& Weyrich, A. S. Targeting phosphodiesterases in anti-platelet therapy. In Handbook of Experimental Pharmacology 225-238, https://doi.org/ 10.1007/978-3-642-29423-5_9 (2012).

45. Zoraghi, R., Kunz, S., Gong, K. \& Seebeck, T. Characterization of TbPDE2A, a novel cyclic nucleotide-specific phosphodiesterase from the protozoan parasite Trypanosoma brucei. J. Biol. Chem. 276, 11559-11566 (2001).

46. Gibbs, C. R. \& Lip, G. Y. Do we still need dipyridamole? Br. J. Clin. Pharmacol. 45, 323-328 (1998).

47. Perlikova, P. \& Hocek, M. Pyrrolo[2,3-d]pyrimidine (7-deazapurine) as a privileged scaffold in design of antitumor and antiviral nucleosides. Med. Res. Rev. 37, 1429-1460 (2017).

48. Geiser, F., Luscher, A., de Koning, H. P., Seebeck, T. \& Maser, P. Molecular pharmacology of adenosine transport in Trypanosoma brucei: P1/P2 revisited. Mol. Pharmacol. 68, 589-595 (2005).

49. Moll, D., Schweinsberg, S., Hammann, C. \& Herberg, F. W. Comparative thermodynamic analysis of cyclic nucleotide binding to protein kinase A. Biol. Chem. 388, 163-172 (2007).

50. Bennett, L. L. Jr. et al. Metabolism and metabolic effects of 2-azahypoxanthine and 2-azaadenosine. Biochem. Pharmacol. 34, 1293-1304 (1985).

51. Mihich, E., Simpson, C. L. \& Mulhern, A. I. Comparative study of the toxicologic effects of 7-deazaadenosine (tubercidin) and 7-deazainosine. Cancer Res. 29, 116-123 (1969).

52. Lorenz, R. et al. Mutations of PKA cyclic nucleotide-binding domains reveal novel aspects of cyclic nucleotide selectivity. Biochem. J. 474, 2389-2403 (2017).

53. Proto, W. R. et al. Trypanosoma brucei metacaspase 4 is a pseudopeptidase and a virulence factor. J. Biol. Chem. 286, 39914-39925 (2011).

54. Herberg, F. W., Taylor, S. S. \& Dostmann, W. R. Active site mutations define the pathway for the cooperative activation of cAMP-dependent protein kinase. Biochemistry 35, 2934-2942 (1996).

55. Kim, J. J. et al. Crystal structure of PKG I:cGMP complex reveals a cGMPmediated dimeric interface that facilitates cGMP-induced activation. Structure 24, 710-720 (2016).

56. Berman, H. M. et al. The cAMP binding domain: an ancient signaling module. Proc. Natl Acad. Sci. USA 102, 45-50 (2005).

57. Dremier, S., Kopperud, R., Doskeland, S. O., Dumont, J. E. \& Maenhaut, C. Search for new cyclic AMP-binding proteins. FEBS Lett. 546, 103-107 (2003).

58. Vass, M. et al. Chemical diversity in the $\mathrm{G}$ protein-coupled receptor superfamily. Trends Pharmacol. Sci. 39, 494-512 (2018). 
59. Schwabe, J. W. R. \& Teichmann, S. A. Nuclear receptors: the evolution of diversity. Sci. STKE 2004, pe4 (2004)

60. Jäger, A. V. et al. Identification of novel cyclic nucleotide binding proteins in Trypanosoma cruzi. Mol. Biochem. Parasitol. 198, 104-112 (2014).

61. Schindler, R. F. R. \& Brand, T. The Popeye domain containing protein family -a novel class of cAMP effectors with important functions in multiple tissues. Prog. Biophys. Mol. Biol. 120, 28-36 (2016).

62. Gjertsen, B. T. et al. Novel (Rp)-cAMPS analogs as tools for inhibition of cAMP-kinase in cell culture. Basal cAMP-kinase activity modulates interleukin-1 beta action. J. Biol. Chem. 270, 20599-20607 (1995)

63. Glisovic, T., Bachorik, J. L., Yong, J. \& Dreyfuss, G. RNA-binding proteins and post-transcriptional gene regulation. FEBS Lett. 582, 1977-1986 (2008).

64. Clayton, C. The regulation of trypanosome gene expression by RNA-binding proteins. PLoS Pathog. 9, e1003680 (2013).

65. Oberholzer, M. et al. Independent analysis of the flagellum surface and matrix proteomes provides insight into flagellum signaling in mammalian-infectious Trypanosoma brucei. Mol. Cell Proteomics. https://doi.org/10.1074/mcp. M111.010538 (2011).

66. Langousis, G. \& Hill, K. L. Motility and more: the flagellum of Trypanosoma brucei. Nat. Rev. Microbiol. 12, 505-518 (2014).

67. McCarty, R. M. \& Bandarian, V. Biosynthesis of pyrrolopyrimidines. Bioorg. Chem. 43, 15-25 (2012).

68. McCarty, R. M. \& Bandarian, V. Deciphering deazapurine biosynthesis: pathway for pyrrolopyrimidine nucleosides toyocamycin and sangivamycin. Chem. Biol. 15, 790-798 (2008).

69. de Koning, H. P., Watson, C. J. \& Jarvis, S. M. Characterization of a nucleoside/proton symporter in procyclic Trypanosoma brucei brucei. J. Biol. Chem. 273, 9486-9494 (1998).

70. Cross, G. A. \& Manning, J. C. Cultivation of Trypanosoma brucei sspp. in semi-defined and defined media. Parasitology 67, 315-331 (1973).

71. Wirtz, E., Leal, S., Ochatt, C. \& Cross, G. A. A tightly regulated inducible expression system for conditional gene knock-outs and dominant-negative genetics in Trypanosoma brucei. Mol. Biochem. Parasitol. 99, 89-101 (1999).

72. Alibu, V. P., Storm, L., Haile, S., Clayton, C. \& Horn, D. A doubly inducible system for RNA interference and rapid RNAi plasmid construction in Trypanosoma brucei. Mol. Biochem. Parasitol. 139, 75-82 (2005).

73. Schimanski, B., Nguyen, T. N. \& Gunzl, A. Highly efficient tandem affinity purification of trypanosome protein complexes based on a novel epitope combination. Eukaryot. Cell 4, 1942-1950 (2005).

74. Veerman, J. et al. Synthesis and evaluation of analogs of the phenylpyridazinone NPD-001 as potent trypanosomal TbrPDEB1 phosphodiesterase inhibitors and in vitro trypanocidals. Bioorg. Med. Chem. 24, 1573-1581 (2016).

75. Hinshaw, B. C., Gerster, J. F., Robins, R. K. \& Townsend, L. B. Pyrrolopyrimidine nucleosides. V. A study on the relative chemical reactivity of the 5-cyano group of the nucleoside antibiotic toyocamycin and desaminotoyocamycin. The synthesis of analogs of sangivamycin. J. Org. Chem. 35, 236-241 (1970).

76. Hastie, C. J., McLauchlan, H. J. \& Cohen, P. Assay of protein kinases using radiolabeled ATP: a protocol. Nat. Protoc. 1, 968-971 (2006).

77. Buechler, Y. J., Herberg, F. W. \& Taylor, S. S. Regulation-defective mutants of type I cAMP-dependent protein kinase. Consequences of replacing arginine 94 and arginine 95. J. Biol. Chem. 268, 16495-16503 (1993).

78. Humphrey, S. J., Karayel, O., James, D. E. \& Mann, M. High-throughput and high-sensitivity phosphoproteomics with the EasyPhos platform. Nat. Protoc. 13, 1897-1916 (2018).
79. Cox, J. \& Mann, M. MaxQuant enables high peptide identification rates individualized p.p.b.-range mass accuracies and proteome-wide protein quantification. Nat. Biotechnol. 26, 1367-1372 (2008).

80. Tyanova, S. et al. The Perseus computational platform for comprehensive analysis of (prote)omics data. Nat. Methods 13, 731-740 (2016).

\section{Acknowledgements}

The authors thank P. Bastin (Pasteur Institute, Paris), A. Bindereif (University of Gießen), C. Dehio (University of Basel), K. Gull (University of Oxford), F. Herberg (University of Kassel), J.H. Leonhardt (LMU Munich), J. Mottram (University of York), E. Pays (Free University of Bruxelles), U. Walter (University of Würzburg), for plasmids or antibodies. A. Mourão (Helmholtz Zentrum Munich) helped with crystal handling and diffraction and T. Wein (LMU Munich) advised on molecular docking. The authors thank U. Manzau and U. Havemann for expert technical assistance with nucleoside and nucleotide syntheses. This work was supported by the University of Munich, Grants DFG 1100/7-1 and BELSPO PAI 6/15 to M.B. and a fellowship from the Science without borders/CNPq program to Y.V.S.

\section{Author contributions}

S.B., Y.V.S., F.S. and M.B. designed research. S.B., Y.V.S., S.K., G.B.G., T.K., J.P., C.B., F.S., J.-W.D. J.B. and I.F. performed research. R.S. wrote a script for analysis. H.-G.G. contributed reagents. S.B., Y.V.S., J.B., E.L., I.F., A.I. and M.B. analyzed data. S.B., Y.V.S. and M.B. wrote the paper.

\section{Additional information}

Supplementary Information accompanies this paper at https://doi.org/10.1038/s41467019-09338-z.

Competing interests: F.S. and H.-G.G. are employed by the BIOLOG Life Science Institute that sells nucleosides and nucleotides that were used in this study or were developed for this study. The other authors declare no competing interests.

Reprints and permission information is available online at http://npg.nature.com/ reprintsandpermissions/

Journal peer review information: Nature Communications thanks Udo Heinemann, Gordon Langsley, and Jeremy Mottram for their contribution to the peer review of this work. Peer reviewer reports are available.

Publisher's note: Springer Nature remains neutral with regard to jurisdictional claims in published maps and institutional affiliations.

Open Access This article is licensed under a Creative Commons Attribution 4.0 International License, which permits use, sharing, adaptation, distribution and reproduction in any medium or format, as long as you give appropriate credit to the original author(s) and the source, provide a link to the Creative Commons license, and indicate if changes were made. The images or other third party material in this article are included in the article's Creative Commons license, unless indicated otherwise in a credit line to the material. If material is not included in the article's Creative Commons license and your intended use is not permitted by statutory regulation or exceeds the permitted use, you will need to obtain permission directly from the copyright holder. To view a copy of this license, visit http://creativecommons.org/ licenses/by/4.0/

() The Author(s) 2019 